\title{
Bounce-averaged Fokker-Planck diffusion equation in non-dipolar magnetic fields with applications to the Dungey magnetosphere
}

\author{
B. Ni, R. M. Thorne, and Q. Ma \\ Department of Atmospheric and Oceanic Sciences, UCLA, CA, USA \\ Correspondence to: B. Ni (bbni@atmos.ucla.edu) \\ Received: 16 November 2011 - Revised: 11 April 2012 - Accepted: 11 April 2012 - Published: 27 April 2012
}

\begin{abstract}
We perform a detailed derivation of the bounceaveraged relativistic Fokker-Planck diffusion equation applicable to arbitrary magnetic field at a constant Roederer $L$. The form of the bounce-averaged diffusion equation is found regardless of details of the mirror geometry, suggesting that the numerical schemes developed for solving the modified two-dimensional (2-D) Fokker-Planck equation in a magnetic dipole should be feasible for similar computation efforts on modeling wave-induced particle diffusion processes in any non-dipolar magnetic field. However, bounce period related terms and bounce-averaged diffusion coefficients are required to be computed in realistic magnetic fields. With the application to the Dungey magnetosphere that is controlled by the intensity of southward interplanetary magnetic field (IMF), we show that with enhanced southward IMF the normalized bounce period related term decreases accordingly, and bounce-averaged diffusion coefficients cover a broader range of electron energy and equatorial pitch angle with a tendency of increased magnitude and peaking at lower energies. The compression of the Dungey magnetosphere can generally produce scattering loss of plasma sheet electrons $<\sim 4 \mathrm{keV}$ and radiation belt electrons $>\sim 100 \mathrm{keV}$ on a timescale shorter than that in a dipolar field, and induce momentum diffusion at high pitch angles closer to $90^{\circ}$. Correspondingly, the strong diffusion rate drops considerably as a product of changes in both the equatorial loss cone and the bounce period. The extent of differences in all the parameters introduced by the southward IMF intensification also becomes larger for a field line with higher equatorial crossing. With the derived general formulism of bounce-averaged diffusion equation for arbitrary 2-D magnetic field, our results confirm the need for the adoption of realistic magnetic fields to perform accurate determination of electron resonant
\end{abstract}

scattering rates and precise multi-dimensional diffusion simulations of magnetospheric electron dynamics.

Keywords. Magnetospheric physics (Auroral phenomena; Magnetospheric configuration and dynamics) - Space plasma physics (Wave-particle interactions)

\section{Introduction}

Resonant wave-particle interactions play an essential role in understanding the dynamics of radiation belt energetic electrons (e.g., Lyons et al., 1972; Summers et al., 1998, 2007a, b, 2008, 2009; O'Brien et al., 2003; Albert, 2004, 2005, 2008; Horne et al., 2005a, b; Meredith et al., 2003, 2006, 2007, 2009a; Miyoshi et al., 2006; Li et al., 2007; Shprits et al., 2008a, b, 2009b, 2011; Thorne et al., 2005, 2007; Zong et al., 2009; Thorne, 2010; Xiao et al., 2009a, 2010b) and plasma sheet source electrons (e.g., Inan et al., 1992; Johnstone et al., 1993; Villalón and Burke, 1995; Horne and Thorne, 2000; Horne et al., 2003; Ni et al., 2008, 2011a, b; Su et al., 2009; Thorne et al., 2010; Tao et al., 2011b). To demonstrate the gyroresonant diffusion processes of magnetospheric electrons due to various plasma waves, quasi-linear theory has been well established assuming that the particle distribution function averaged over space changes slowly on time scales associated with the motion of the waves (Kennel and Engelmann, 1966). Omitting particle trapping and highly nonlinear effects, quasi-linear theory describes stochastic interactions between a succession of small amplitude waves with random phase and charged particles in terms of a diffusion equation and quasi-linear diffusion coefficients.

To justify the applicability of quasi-linear theory, Albert (2010) recently used two formulations of diffusion which are conceptually different and demonstrated that suitably 
averaging the monochromatic diffusion coefficients over chorus frequency and wave normal angle parameters can favourably reproduce the full broadband quasi-linear results. Further verification of quasi-linear theory has been implemented using test particle simulations, e.g., by Bortnik et al. (2008) and Tao et al. (2011a) for whistler-mode chorus, by Liu et al. (2010) for electromagnetic ion cyclotron (EMIC) waves, and by Bortnik and Thorne (2010) for magnetosonic waves. All those studies concluded that the diffusion coefficients obtained by the test particle computations agree well with the results derived from quasi-linear theory when wave amplitudes are small. In addition, quasi-linear diffusion theory has been successfully used to provide an effective overall description of wave-induced resonant diffusion. For instance, quasi-linear scattering has been remarkably effective at quantifying both the rates of microburst scattering loss by chorus (Thorne et al., 2005) and the rates of local acceleration to relativistic energies (e.g., Horne et al., 2005a; Thorne et al., 2007). Multi-dimensional simulations developed to solve numerically the Fokker-Planck diffusion equation (e.g., Beutier and Boscher, 1995; Bourdarie et al., 1996; Shprits et al., 2006a, 2009b; Li et al., 2007; Tao et al., 2008, 2009; Xiao et al., 2009a, 2010b; Albert et al., 2009; Subbotin et al., 2010) also showed a good correspondence of model results with observations of radiation belt electrons (e.g., Albert et al., 2009; Su et al., 2010a; Shprits et al., 2011; Subbotin et al., 2011) and inner plasma sheet electrons (e.g., Su et al., 2009; Thorne et al., 2010; Tao et al., 2011b).

However, all those diffusion simulations have concentrated on a modified Fokker-Planck equation bounceaveraged in a dipolar geometry with bounce period and quasi-linear diffusion coefficients evaluated in the same magnetic field topology. In contrast, during geomagnetically disturbed periods (e.g., magnetic storms and substorms), the realistic ambient magnetic field in the magnetosphere of $L>\sim 5$ can deviate considerably from a dipole representation both in configuration and field strength. Recently, Orlova and Shprits (2010) and Ni et al. (2011c) have reported respectively that bounce-averaged quasi-linear scattering rates of radiation belt relativistic electrons and plasma sheet source electrons depend strongly on the adoption of ambient magnetic field model and the level of geomagnetic activity. However, those studies solely focused on the effect of non-dipolar magnetic fields on the determination of quasi-linear scattering rates and loss timescales of electrons. While diffusion coefficients are important to evaluate wave-induced particle diffusion effects, they are just a large portion of input parameters required to solve numerically the Fokker-Planck diffusion equation that also needs to consider the geometry of ambient magnetic field for outputting the evolution of particle distribution that we are highly interested in. Although there were a few earlier studies exploring particle motion and diffusion in dipolar and non-dipolar magnetic mirror geometries (e.g., MacDonald and Walt, 1961; Wentworth, 1963; Schulz, 1976; Schulz and Chen, 1995), there is no detailed investi- gation of bounce-averaging the localized Fokker-Planck diffusion equation in arbitrary magnetic field and of the effect of non-dipolar fields on numerical simulation of the modified Fokker-Planck diffusion equation, which will be the subject of the present study.

The remainder of this paper is organized as follows. In Sect. 2 we give a detailed derivation of the bounceaveraged Fokker-Planck diffusion equation applicable to arbitrary magnetic field. We identify the differences between diffusion simulations in dipolar and non-dipolar magnetic fields. To explore the importance of more realistic magnetic fields on understanding the highly dynamic nature of magnetospheric electrons and the role of resonant wave-particle interactions, we apply the generalized diffusion formulism to the Dungey magnetosphere in Sect. 3. At two representative magnetic field lines with equatorial crossings at 6 and 9 Earth radii, we evaluate bounce period related term $\left(S_{0}\right)$, bounceaveraged quasi-linear diffusion coefficients $\left(\left\langle D_{\alpha \alpha}\right\rangle,\left\langle D_{\alpha p}\right\rangle\right.$, and $\left.\left(D_{p p}\right\rangle\right)$, and strong diffusion rate $\left(D_{\mathrm{SD}}\right)$ for quantitative comparisons with those obtained using a dipolar field. We discuss the results in Sect. 4 and finally summarize this study in Sect. 5.

\section{Bounce-averaging of Fokker-Planck equation in arbi- trary magnetic field}

According to Summers (2005) and Summers and Ni (2008), after ignoring radial diffusion, an alternate and common form of the localized two-dimensional (2-D) relativistic FokkerPlanck diffusion equation for gyrophase-averaged phase space density (PSD) $f$ can be described as

$$
\begin{aligned}
\frac{\partial f}{\partial t}= & \frac{1}{\sin \alpha} \frac{\partial}{\partial \alpha}\left(D_{\alpha \alpha} \sin \alpha \frac{\partial f}{\partial \alpha}\right)+\frac{1}{\sin \alpha} \frac{\partial}{\partial \alpha}\left(D_{\alpha p} \sin \alpha \frac{\partial f}{\partial p}\right) \\
& +\frac{1}{p^{2}} \frac{\partial}{\partial p}\left(p^{2} D_{p \alpha} \frac{\partial f}{\partial \alpha}\right)+\frac{1}{p^{2}} \frac{\partial}{\partial p}\left(p^{2} D_{p p} \frac{\partial f}{\partial p}\right),
\end{aligned}
$$

where $t$ denotes time, $\alpha$ is the local pitch angle; $p=\gamma m v$ is the particle momentum where $v$ is the particle velocity and $m$ is the rest mass; $\gamma=\left(1-v^{2} / c^{2}\right)^{-1 / 2}$ is the Lorentz factor with $c$ as the speed of light. $D_{\alpha \alpha}, D_{\alpha p}=D_{p \alpha}, D_{p p}$ are local rates of pitch angle diffusion, mixed diffusion, and momentum diffusion, respectively, which are defined as:

$D_{\alpha \alpha}=(\Delta \alpha)^{2} / \Delta t$,

$D_{\alpha p}=(\Delta \alpha \Delta p) / \Delta t$,

$D_{p p}=(\Delta p)^{2} / \Delta t$

Both diffusion Eq. (1) and diffusion rate formula (2)-(4) are derived for a uniform background magnetic field, i.e., at a 
given point in space. In order to apply them to a magnetic mirror geometry such as the Earth's magnetic field, Eqs. (1)(4) must be bounce-averaged, that is, averaged over particle bounce orbits.

Bounce-averaging of local diffusion coefficients has been well established in a dipolar field by a number of previous studies (e.g., Lyons et al., 1972; Glauert and Horne, 2005; Shprits et al., 2006b; Summers et al., 2007a). For arbitrary magnetic mirror geometry, the general form for bounceaveraged quasi-linear diffusion coefficients can be written as

$\left\langle D_{\alpha \alpha}\right\rangle=\frac{1}{\tau_{\mathrm{B}}} \int_{0}^{\tau_{\mathrm{B}}} D_{\alpha \alpha}(\alpha)\left(\frac{\partial \alpha_{\mathrm{eq}}}{\partial \alpha}\right)^{2} d t$,

$\left\langle\frac{D_{\alpha p}}{p}\right\rangle=\frac{1}{\tau_{\mathrm{B}}} \int_{0}^{\tau_{\mathrm{B}}} \frac{D_{\alpha p}(\alpha)}{p}\left(\frac{\partial \alpha_{\mathrm{eq}}}{\partial \alpha}\right) d t$,

$\left\langle\frac{D_{p p}}{p^{2}}\right\rangle=\frac{1}{\tau_{\mathrm{B}}} \int_{0}^{\tau_{\mathrm{B}}} \frac{D_{p p}(\alpha)}{p^{2}} d t$,

where $\alpha_{\mathrm{eq}}$ is the equatorial pitch angle, and $\tau_{\mathrm{B}}$ is the energy dependent bounce period given by:

$\tau_{\mathrm{B}}=\oint_{S} \frac{d s}{v \cos \alpha}=\frac{2}{v} \int_{\lambda_{\mathrm{m} 1}}^{\lambda_{\mathrm{m} 2}} \frac{(\partial s / \partial \lambda)}{\cos \alpha} d \lambda$.

Here $d s$ is the element of arc length along the particle bounce trajectory, $\lambda$ is the magnetic latitude, and $\lambda_{\mathrm{m} 1}$ and $\lambda_{\mathrm{m} 2}$ are the mirror latitude of particles on the Southern and Northern Hemisphere, respectively, dependent on the field line configuration and the field strength of adopted magnetic field model. Note that we have scaled $D_{\alpha p}$ and $D_{p p}$ by $p$ and $p^{2}$ respectively to obtain the conventional units of $\mathrm{s}^{-1}$ for quasilinear diffusion coefficients. For arbitrary magnetic field line that lies in a plane perpendicular to the magnetic equator, i.e., a two-dimensional field line topology, which is a good approximation under most geomagnetic conditions, Eq. (8) can be rewritten as

$\tau_{\mathrm{B}}=\frac{2}{v} \int_{\lambda_{\mathrm{m} 1}}^{\lambda_{\mathrm{m} 2}} \frac{\sqrt{r^{2}+(\partial r / \partial \lambda)^{2}}}{\cos \alpha} d \lambda$,

where $r$ is the radial distance to the Earth's center. In addition, conservation of the first adiabatic invariant provides a general relationship between $\alpha_{\mathrm{eq}}$ and $\alpha$, that is,

$\sin ^{2} \alpha_{\text {eq }} / \sin ^{2} \alpha=B_{\text {eq }} / B$,

with $B_{\text {eq }}$ and $B$ as the equatorial and local magnetic field strength, respectively. From Eq. (10) we further obtain the derivative:

$\frac{\partial \alpha_{\mathrm{eq}}}{\partial \alpha}=\left(\frac{B_{\mathrm{eq}}}{B}\right) \frac{\sin \alpha \cos \alpha}{\sin \alpha_{\mathrm{eq}} \cos \alpha_{\mathrm{eq}}}=\frac{\tan \alpha_{\mathrm{eq}}}{\tan \alpha}$.
Taking into account that $d t=d s /(v \cos \alpha)$ and putting Eqs. (9) and (11) into Eqs. (5)-(7), we have:

$\left\langle D_{\alpha \alpha}\right\rangle=\frac{\int_{\lambda_{\mathrm{m} 1}}^{\lambda_{\mathrm{m} 2}} \frac{D_{\alpha \alpha}(\alpha)}{\cos \alpha}\left(\frac{\tan \alpha_{\mathrm{eq}}}{\tan \alpha}\right)^{2} \sqrt{r^{2}+\left(\frac{\partial r}{\partial \lambda}\right)^{2}} d \lambda}{\int_{\lambda \mathrm{m} 1}^{\lambda_{\mathrm{m} 2}} \sec \alpha \sqrt{r^{2}+\left(\frac{\partial r}{\partial \lambda}\right)^{2}} d \lambda}$,

$\left\langle\frac{D_{\alpha p}}{p}\right\rangle=\frac{\int_{\lambda_{\mathrm{m} 1} 1}^{\lambda_{\mathrm{m}}} \frac{D_{\alpha p}(\alpha)}{p \cos \alpha} \frac{\tan \alpha_{\mathrm{eq}}}{\tan \alpha} \sqrt{r^{2}+\left(\frac{\partial r}{\partial \lambda}\right)^{2}} d \lambda}{\int_{\lambda_{\mathrm{m} 1}}^{\lambda_{\mathrm{m} 2}} \sec \alpha \sqrt{r^{2}+\left(\frac{\partial r}{\partial \lambda}\right)^{2}} d \lambda}$,

$\left\langle\frac{D_{p p}}{p^{2}}\right\rangle=\frac{\int_{\lambda_{\mathrm{m} 1}}^{\lambda_{\mathrm{m} 2}} \frac{D_{p p}(\alpha)}{p^{2} \cos \alpha} \sqrt{r^{2}+\left(\frac{\partial r}{\partial \lambda}\right)^{2}} d \lambda}{\int_{\lambda_{\mathrm{m} 1}}^{\lambda_{\mathrm{m} 2}} \sec \alpha \sqrt{r^{2}+\left(\frac{\partial r}{\partial \lambda}\right)^{2}} d \lambda}$.

Equations (12)-(14) represent the general formula for evaluation of bounce-averaged quasi-linear diffusion coefficients in any 2-D magnetic field configuration.

Now we carry out bounce-averaging of localized FokkerPlanck diffusion Eq. (1) over a particle bounce orbit. After bounce-averaging, the first term on the right-hand side becomes:

$$
\begin{aligned}
& \frac{1}{\tau_{\mathrm{B}}} \int_{0}^{\tau_{\mathrm{B}}} \frac{1}{\sin \alpha} \frac{\partial}{\partial \alpha}\left(D_{\alpha \alpha} \sin \alpha \frac{\partial f}{\partial \alpha}\right) d t \\
& =\frac{1}{\int_{\lambda_{\mathrm{m} 2}}\left(\frac{\partial s}{\partial \lambda}\right) \sec \alpha d \lambda \lambda_{\mathrm{m} 1}} \int^{\lambda_{\mathrm{m} 2}} \csc \alpha \sec \alpha \\
& \\
& \quad\left(\frac{\partial s}{\partial \lambda}\right) \frac{\partial \alpha_{\mathrm{eq}}}{\partial \alpha} \frac{\partial}{\partial \alpha_{\mathrm{eq}}}\left(D_{\alpha \alpha} \sin \alpha \frac{\partial \alpha_{\mathrm{eq}}}{\partial \alpha} \frac{\partial f}{\partial \alpha_{\mathrm{eq}}}\right) d \lambda .
\end{aligned}
$$

Taking Eqs. (11) and (10) into Eq. (15), we have:

$$
\begin{aligned}
& \frac{1}{\tau_{\mathrm{B}}} \int_{0}^{\tau_{\mathrm{B}}} \frac{1}{\sin \alpha} \frac{\partial}{\partial \alpha}\left(D_{\alpha \alpha} \sin \alpha \frac{\partial f}{\partial \alpha}\right) d t \\
& =\frac{1}{\sin \alpha_{\mathrm{eq}} \cos \alpha_{\mathrm{eq}} \int_{\lambda_{\mathrm{m} 1}}^{\lambda_{\mathrm{m} 2}}\left(\frac{\partial s}{\partial \lambda}\right) \sec \alpha d \lambda} \frac{\partial}{\partial \alpha_{\mathrm{eq}}}
\end{aligned}
$$

Ann. Geophys., 30, 733-750, 2012 


$$
\begin{aligned}
& {\left[\int_{\lambda_{\mathrm{m} 1}}^{\lambda_{\mathrm{m} 2}}\left(\frac{B_{\mathrm{eq}}}{B}\right)^{2}\left(\frac{\partial s}{\partial \lambda}\right) D_{\alpha \alpha} \frac{\sin ^{2} \alpha \cos \alpha}{\sin \alpha_{\mathrm{eq}} \cos \alpha_{\mathrm{eq}}} d \lambda \frac{\partial f}{\partial \alpha_{\mathrm{eq}}}\right] } \\
= & \frac{1}{\sin \alpha_{\mathrm{eq}} \cos \alpha_{\mathrm{eq}} \int_{\lambda_{\mathrm{m} 1}}^{\lambda_{\mathrm{m} 2}}\left(\frac{\partial s}{\partial \lambda}\right) \sec \alpha d \lambda} \frac{\partial \alpha_{\mathrm{eq}}}{\left.\sin \alpha_{\mathrm{eq}} \cos \alpha_{\mathrm{eq}} \int_{\lambda_{\mathrm{m} 1}}^{\lambda_{\mathrm{m} 2}} \frac{D_{\alpha \alpha}}{\cos \alpha}\left(\frac{\tan \alpha_{\mathrm{eq}}}{\tan \alpha}\right)^{2}\left(\frac{\partial s}{\partial \lambda}\right) d \lambda \frac{\partial f}{\partial \alpha_{\mathrm{eq}}}\right]} \\
= & \frac{1}{\lambda_{\mathrm{m} 2}} \frac{\partial}{\partial \alpha_{\mathrm{eq}}} \\
& {\left[\begin{array}{l}
\sin \alpha_{\mathrm{eq}} \cos \alpha_{\mathrm{eq}} \int_{\lambda_{\mathrm{m} 1}} \\
\sin \alpha_{\mathrm{eq}} \cos \alpha_{\mathrm{eq}} \int_{\lambda_{\mathrm{m} 1}}^{\lambda_{\mathrm{m} 2}}\left(\frac{\partial s}{\partial \lambda}\right) \sec \alpha d \lambda
\end{array}\right] }
\end{aligned}
$$

Setting

$S_{0}\left(\alpha_{\mathrm{eq}}\right)=\int_{\lambda_{\mathrm{m} 1}}^{\lambda_{\mathrm{m} 2}}\left(\frac{\partial s}{\partial \lambda}\right) \sec \alpha d \lambda=\int_{\lambda_{\mathrm{m} 1}}^{\lambda_{\mathrm{m} 2}} \sec \alpha \sqrt{r^{2}+\left(\frac{\partial r}{\partial \lambda}\right)^{2}} d \lambda$,

we obtain:

$$
\begin{gathered}
\frac{1}{\tau_{\mathrm{B}}} \int_{0}^{\tau_{\mathrm{B}}} \frac{1}{\sin \alpha} \frac{\partial}{\partial \alpha}\left(D_{\alpha \alpha} \sin \alpha \frac{\partial f}{\partial \alpha}\right) d t=\frac{1}{S_{0} \sin \alpha_{\mathrm{eq}} \cos \alpha_{\mathrm{eq}}} \frac{\partial}{\partial \alpha_{\mathrm{eq}}} \\
{\left[S_{0} \sin \alpha_{\mathrm{eq}} \cos \alpha_{\mathrm{eq}}\left\langle D_{\alpha \alpha}\right\rangle \frac{\partial f}{\partial \alpha_{\mathrm{eq}}}\right] .}
\end{gathered}
$$

Similarly, bounce-averaging the second to fourth terms on the right-hand side yields:

$$
\begin{aligned}
& \frac{1}{\tau_{\mathrm{B}}} \int_{0}^{\tau_{\mathrm{B}}} \frac{1}{\sin \alpha} \frac{\partial}{\partial \alpha}\left(D_{\alpha p} \sin \alpha \frac{\partial f}{\partial p}\right) d t= \\
& \frac{1}{S_{0} \sin \alpha_{\mathrm{eq}} \cos \alpha_{\mathrm{eq}}} \frac{\partial}{\partial \alpha_{\mathrm{eq}}}\left[S_{0} \sin \alpha_{\mathrm{eq}} \cos \alpha_{\mathrm{eq}}\left\langle D_{\alpha p}\right\rangle \frac{\partial f}{\partial p}\right],
\end{aligned}
$$

$$
\frac{1}{\tau_{\mathrm{B}}} \int_{0}^{\tau_{\mathrm{B}}} \frac{1}{p^{2}} \frac{\partial}{\partial p}\left(p^{2} D_{p \alpha} \frac{\partial f}{\partial \alpha}\right) d t=\frac{1}{p^{2}} \frac{\partial}{\partial p}\left[p^{2}\left\langle D_{p \alpha}\right\rangle \frac{\partial f}{\partial \alpha_{\mathrm{eq}}}\right],
$$

$$
\frac{1}{\tau_{\mathrm{B}}} \int_{0}^{\tau_{\mathrm{B}}} \frac{1}{p^{2}} \frac{\partial}{\partial p}\left(p^{2} D_{p p} \frac{\partial f}{\partial p}\right) d t=\frac{1}{p^{2}} \frac{\partial}{\partial p}\left[p^{2}\left\langle D_{p p}\right\rangle \frac{\partial f}{\partial p}\right] .
$$

Finally, we can express the bounce-averaged 2-D relativistic Fokker-Planck diffusion equation for particle PSD $(f)$ evolution as a function of equatorial pitch angle $\left(\alpha_{\mathrm{eq}}\right)$ and particle momentum $(p)$ in a general form,

$$
\begin{aligned}
\frac{\partial f}{\partial t}= & \frac{1}{S_{0} \sin \alpha_{\mathrm{eq}} \cos \alpha_{\mathrm{eq}}} \frac{\partial}{\partial \alpha_{\mathrm{eq}}} \\
& {\left[S_{0} \sin \alpha_{\mathrm{eq}} \cos \alpha_{\mathrm{eq}}\left(\left\langle D_{\alpha \alpha}\right\rangle \frac{\partial f}{\partial \alpha_{\mathrm{eq}}}+\left\langle D_{\alpha p}\right\rangle \frac{\partial f}{\partial p}\right)\right] } \\
& +\frac{1}{p^{2}} \frac{\partial}{\partial p}\left[p^{2}\left(\left\langle D_{p \alpha}\right\rangle \frac{\partial f}{\partial \alpha_{\mathrm{eq}}}+\left\langle D_{p p}\right\rangle \frac{\partial f}{\partial p}\right)\right] .
\end{aligned}
$$

Equation (22) demonstrates that at a constant Roederer $L$ (Roederer, 1970) the bounce-averaged Fokker-Planck diffusion equation has the similar expression for arbitrary magnetic field except that the bounce period related term $S_{0}$ and bounce-averaged quasi-linear diffusion coefficients $\left\langle D_{\alpha \alpha}\right\rangle$, $\left\langle D_{\alpha p}\right\rangle$, and $\left\langle D_{p p}\right\rangle$ are required to be evaluated in correspondence with adopted magnetic field model. It is expected that use of different magnetic field models for Fokker-Planck diffusion simulations will produce different results regarding the evolution of magnetospheric particles. In general, a model that represents the ambient magnetic field more accurately tends to help us better understand the particle dynamics in the realistic geo-space environment. Also, the uniformity in equation expression suggests that the numerical schemes developed for solving the modified Fokker-Planck equation in a magnetic dipole should be feasible for similar computation efforts on modeling wave-induced particle diffusion processes using any non-dipolar magnetic field.

\section{Application to the Dungey magnetosphere}

To illustrate how the terms associated with the general bounce-averaged 2-D Fokker-Planck diffusion Eq. (22) change with the adoption of magnetic field model, we apply the Dungey magnetic fields (Dungey, 1961) in the following study.

\subsection{The Dungey magnetic field model}

To take into account the effect of southward interplanetary magnetic field (IMF) on the configuration and strength of the ambient magnetic field, the Dungey magnetic field model consists of the geomagnetic dipolar field plus a uniform southward magnetic field and thus has three components as follows (e.g., Chen et al., 1993; Schulz, 1998),

$$
\begin{aligned}
& B_{\mathrm{r}}=-\left(\frac{2 M}{r^{3}}+B_{\mathrm{z}, 0}\right) \sin \lambda, \\
& B_{\lambda}=\left(\frac{M}{r^{3}}-B_{\mathrm{z}, 0}\right) \cos \lambda, \\
& B_{\varphi}=0 .
\end{aligned}
$$




$$
r_{0}=6 R_{E}
$$

(a)

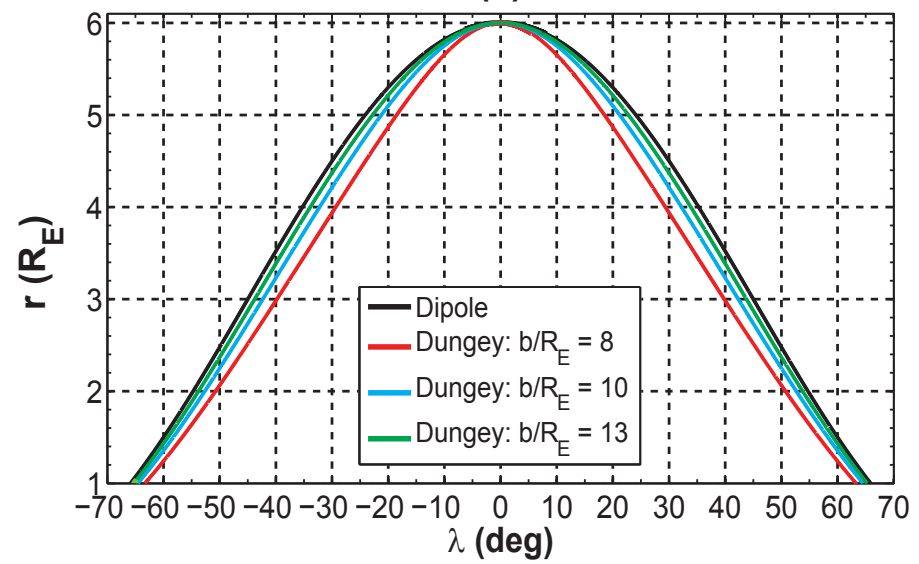

(b)

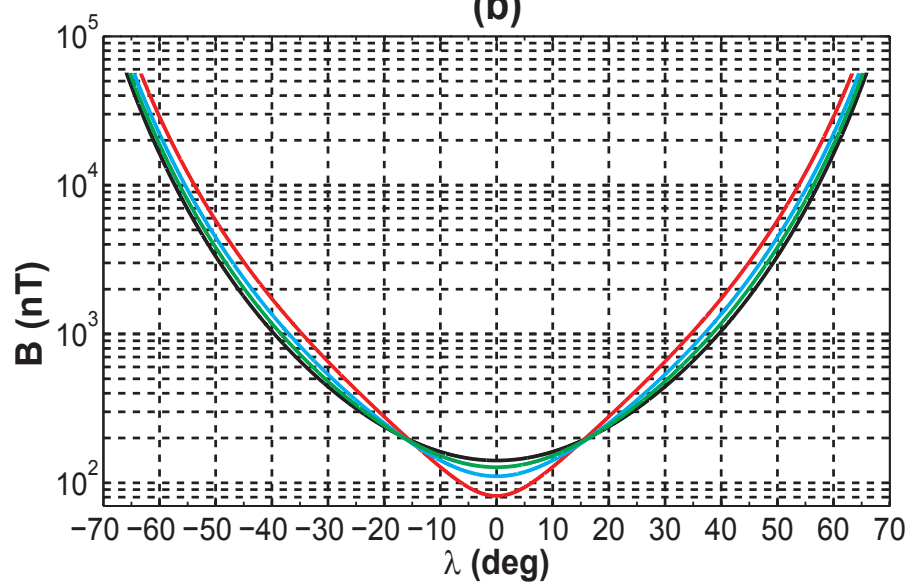

$r_{0}=9 R_{E}$

(c)

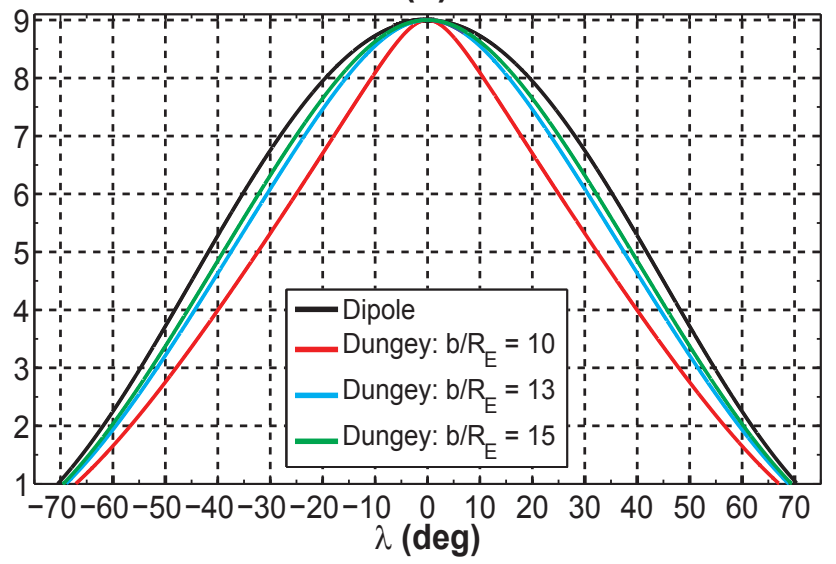

(d)

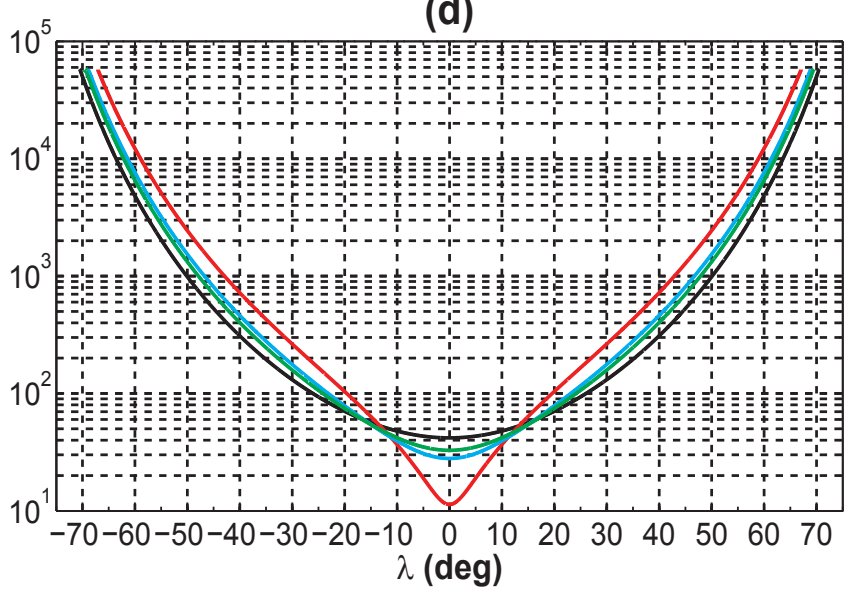

Fig. 1. Field line configuration and magnetic field intensity of the Dungey magnetosphere for the field lines with two specific equatorial crossings of (a), (b) $r_{0}=6 R_{\mathrm{E}}$ for $b / R_{\mathrm{E}}=\infty$ (dipole), 13, 10, and 8, and of (c), (d) $r_{0}=9 R_{\mathrm{E}}$ for $b / R_{\mathrm{E}}=\infty$ (dipole), 15, 13, and 10.

Here $M$ is the magnetic moment of the dipole, $r$ is the radial distance, $B_{\mathrm{z}, 0}$ is a uniform magnetic field normal to the dipole's equatorial plane, directed southward and parallel to $M, \lambda$ is the magnetic latitude and $\varphi$ is the magnetic longitude. The total ambient magnetic field intensity is given

$$
\begin{aligned}
B & =\left(B_{\mathrm{r}}^{2}+B_{\lambda}^{2}\right)^{1 / 2} \\
& =\frac{M}{r^{3}}\left\{\left[1-\left(\frac{r}{b}\right)^{3}\right]^{2}+3\left[1+2\left(\frac{r}{b}\right)^{3}\right] \sin ^{2} \lambda\right\}^{1 / 2}
\end{aligned}
$$

and the equation of magnetic field line is

$r=r_{0}\left[1+\frac{1}{2}\left(\frac{r}{b}\right)^{3}\right] \cos ^{2} \lambda$

where $b=\left(M / B_{\mathrm{z}, 0}\right)^{1 / 3}$ is the parameter describing the compression level of the Earth's dipole magnetic field, and $r_{0}$ is the radial distance in the equatorial plane. Equation (27) can be rewritten as

$\frac{1}{b^{3}} r^{3}-\frac{2}{r_{0} \cos ^{2} \lambda} r+2=0$,

from which $r$ can be determined once $B_{\mathrm{z}, 0}$ and $\lambda$ are given. The Dungey magnetic field reduces to the geomagnetic dipolar field when $b$ is infinity or $B_{\mathrm{z}, 0}=0 \mathrm{nT}$.

Figure 1 shows the field line configuration and magnetic field intensity of the Dungey magnetosphere corresponding to two specific equatorial crossings at $r_{0}=6 R_{\mathrm{E}}\left(R_{\mathrm{E}}\right.$ is the Earth radius) for $b / R_{\mathrm{E}}=\infty$ (dipole), 13, 10, and 8 , and at $r_{0}=9 R_{\mathrm{E}}$ for $b / R_{\mathrm{E}}=\infty$ (dipole), 15, 13, and 10 . Clearly, as $b$ decreases, i.e., the southward IMF intensifies, the Dungey magnetic field is increasingly compressed with the footprint latitude $\lambda_{\mathrm{f}}$ at the Earth's surface decreasing accordingly. Specifically, at $r_{0}=6 R_{\mathrm{E}} \lambda_{\mathrm{f}}=65.9^{\circ}, 65.3^{\circ}, 64.6^{\circ}$, $63.3^{\circ}$ for $b / R_{\mathrm{E}}=\infty, 13,10$, and 8 , respectively; at $r_{0}=9 R_{\mathrm{E}}$ $\lambda_{\mathrm{f}}=70.5^{\circ}, 69.5^{\circ}, 68.9^{\circ}, 67.1^{\circ}$ for $b / R_{\mathrm{E}}=\infty, 15,13$, and 10 , respectively. Magnetic field strength varies in a distinct 
manner, decreasing at low latitudes (within $\sim 15^{\circ}$ ) near the magnetic equator but increasing at higher latitudes as $b$-value decreases. In addition, the differences in the Dungey magnetic field are enhanced for magnetic field lines with larger equatorial crossing. Under the same southward IMF condition, say, $b / R_{\mathrm{E}}=10$, the field line geometry for $r_{0}=9 R_{\mathrm{E}}$ deviates farther away from a dipole; the corresponding magnetic field amplitude can decrease by a factor of $\sim 4$ near the equator and increase by a factor of $\sim 2$ at higher latitudes, more pronounced than that for $r_{0}=6 R_{\mathrm{E}}$.

\subsection{Bounce period related term $S_{0}$}

The motion of particles trapped in a magnetic mirror geometry almost invariably leads to a complex integral expression as shown, e.g., in Eq. (17). In a dipolar field, the normalized $S_{0}$, as a quarter-bounce integral, has been investigated in detail by Lenchek et al. (1961) and Davidson (1976) and recently revisited by Orlova and Shprits (2011). Two good empirical approximations of $S_{0}$ in terms of $\sin \alpha_{\mathrm{eq}}$,

$S_{0} \approx 1.30-0.56 \sin \alpha_{\mathrm{eq}}$

with an error within $4.5 \%$ and

$S_{0} \approx 1.38-0.32\left[\sin \alpha_{\mathrm{eq}}+\left(\sin \alpha_{\mathrm{eq}}\right)^{1 / 2}\right]$

with an error within $1 \%$, have been widely adopted for multidimensional diffusion simulations in a dipolar field (e.g., Shprits et al., 2006a, 2009a, 2011; Li et al., 2007; Tao et al., 2008, 2009; Xiao et al., 2009a, 2010b; Albert et al., 2009; Subbotin et al., 2010, 2011). Readers are referred to Davidson (1976) and Orlova and Shprits (2011) for more accurate approximations of $S_{0}$ as the quarter-bounce integral in a dipolar field.

Defining $S_{0}$ as a half-bounce integral for generalization, Eq. (17) can be applied to arbitrary 2-D magnetic field. Since the Dungey magnetic field is axisymmetric and $S_{0}$ appears on both the numerator and the denominator of Eq. (22), it is convenient to compute $S_{0}$ as a quarter-bounce integral in this study,

$S_{0}\left(\alpha_{\mathrm{eq}}\right)=\int_{r_{\mathrm{m}}}^{r_{0}} \sec \alpha \sqrt{\left(r \frac{\partial \lambda}{\partial r}\right)^{2}+1} d r$,

where the radial distance at the mirror latitude $r_{\mathrm{m}}$ is a function of $\alpha_{\text {eq }}$, determined from a ninth-order polynomial equation

$$
\begin{gathered}
\left\{\frac{\left[1-\left(r_{0} / b\right)^{3}\right]^{2}}{r_{0}^{6} b^{3} \sin ^{4} \alpha_{0}}-b^{-9}\right\} r_{\mathrm{m}}^{9}+\left\{\frac{2\left[1-\left(r_{0} / b\right)^{3}\right]^{2}}{r_{0}^{6} \sin ^{4} \alpha_{0}}-6 b^{-6}\right\} r_{\mathrm{m}}^{6} \\
+\frac{12}{r_{0} b^{3}} r_{\mathrm{m}}^{4}-\frac{12}{b^{3}} r_{\mathrm{m}}^{3}+\frac{6}{r_{0}} r_{\mathrm{m}}-8=0
\end{gathered}
$$

for given values of $r_{0}$ and $b$; $(\partial \lambda / \partial r)^{2}$ can be obtained by:

$$
(\partial \lambda / \partial r)^{2}=\frac{4 r\left[\frac{3 r^{2}}{b^{3}}-\frac{2+(r / b)^{3}}{r}\right]^{2}}{16 r_{0}\left[2+\left(\frac{r}{b}\right)^{2}\right]^{3}\left[2+\left(\frac{r}{b}\right)^{2}-\frac{2 r}{r_{0}}\right]} .
$$

Equations (32) and (33) are easily derived from Eqs. (10), (26), and (27).

Figure $2 \mathrm{a}$ and $\mathrm{b}$ shows the mirror radial distance $r_{\mathrm{m}}$ (determined from Eq. 32) and the mirror latitude $\lambda_{\mathrm{m}}$ (determined from Eq. 27) as a function of $\alpha_{\mathrm{eq}}$ for $r_{0}=6 R_{\mathrm{E}}$ with $b / R_{\mathrm{E}}=\infty$ (dipole), 13, 10, and 8. As $b$ increases, $r_{\mathrm{m}}$ decreases but $\lambda_{\mathrm{m}}$ increases with the largest difference occurring at intermediate $\alpha_{\text {eq }}$ of $\sim 15^{\circ}-55^{\circ}$ compared to the dipole results. Variation of mirror latitude directly modifies the spatial extent of the resonance zone where resonance between wave and particles can take place (e.g., Ni and Summers, $2010 \mathrm{a}, \mathrm{b})$. The bounce period related term $S_{0}$, calculated using Eq. (31) and normalized by $r_{0}$ (thus dimensionless), is shown in Fig. 2c as color-coded solid curves for the four values of $b$. As expected, $S_{0}$, and correspondingly the bounce period of any particle, decreases when the southward IMF intensifies, mainly due to the compression of magnetic field line with a shorter length. Compared to the results in a dipolar field, the degree of decrease in $S_{0}$ increases considerably with equatorial pitch angle, varying from $\sim 1 \%$ at $\alpha_{\text {eq }} \approx 0^{\circ}$ to $>50 \%$ at $\alpha_{\mathrm{eq}} \approx 90^{\circ}$. Based upon the previous studies (e.g., Lenchek et al., 1961; Schulz and Lanzerotti, 1974; Davidson, 1976; Orlova and Shprits, 2011), we have adopted a fifthorder polynomial of $\sqrt{\sin \alpha_{\text {eq }}}$,

$$
\begin{aligned}
S_{0}\left(\alpha_{\mathrm{eq}}\right)= & a_{5}\left(\sqrt{\sin \alpha_{\mathrm{eq}}}\right)^{5}+a_{4}\left(\sqrt{\sin \alpha_{\mathrm{eq}}}\right)^{4}+a_{3}\left(\sqrt{\sin \alpha_{\mathrm{eq}}}\right)^{3} \\
& +a_{2}\left(\sqrt{\sin \alpha_{\mathrm{eq}}}\right)^{2}+a_{1}\left(\sqrt{\sin \alpha_{\mathrm{eq}}}\right)+a_{0},
\end{aligned}
$$

to establish an empirical analytic representation of the normalized $S_{0}$ values with outstanding accuracy, which can be practical for multi-dimensional diffusion simulations in the Dungey magnetic field that will be investigated in detail in a companion paper by Ma et al. (2012). The fitted values of six polynomial coefficients $\left(a_{0}-a_{5}\right)$ are tabulated on the top half of Table 1 for each $b$-value at $r_{0}=6 R_{\mathrm{E}}$, and these approximation formulae are also plotted as dashed curves in Fig. 2c. They overlap very well with the accurate numerical results shown as solid curves, demonstrating an excellent empirical description of bounce period related term in the Dungey magnetosphere. This consistency is further justified by the errors associated with the fitted polynomials, as shown in Fig. 2d, indicating a maximum value of below $0.5 \%$ for all $\alpha_{\mathrm{eq}}$. The corresponding results for $r_{0}=9 R_{\mathrm{E}}$ with $b / R_{\mathrm{E}}=\infty$ (dipole), 15, 13, and 10 are illustrated in Fig. 3. Variations of $r_{\mathrm{m}}, \lambda_{\mathrm{m}}$ and normalized $S_{0}$ show profiles with respect to $\alpha_{\mathrm{eq}}$ and $b$ in similar to that for $r_{0}=6 R_{\mathrm{E}}$, except that the extents of differences compared to the dipolar results are larger. The fitted parameters of $a_{0}-a_{5}$ are listed on the bottom half of Table 1. 
(a)

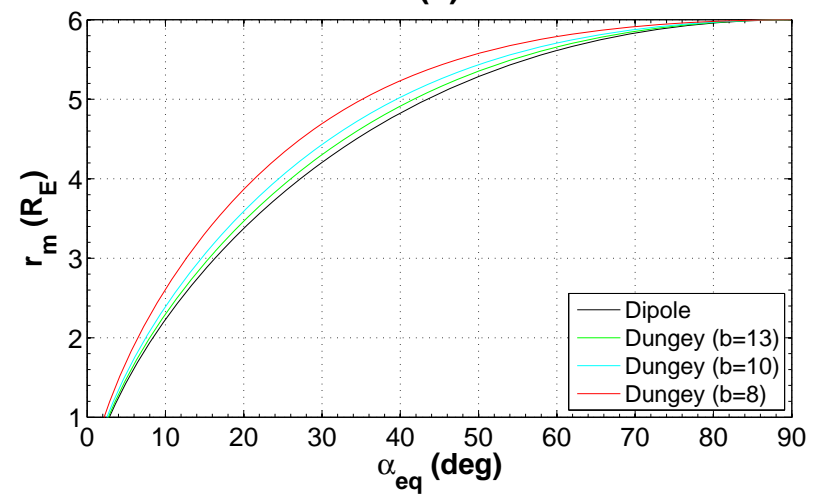

(c)

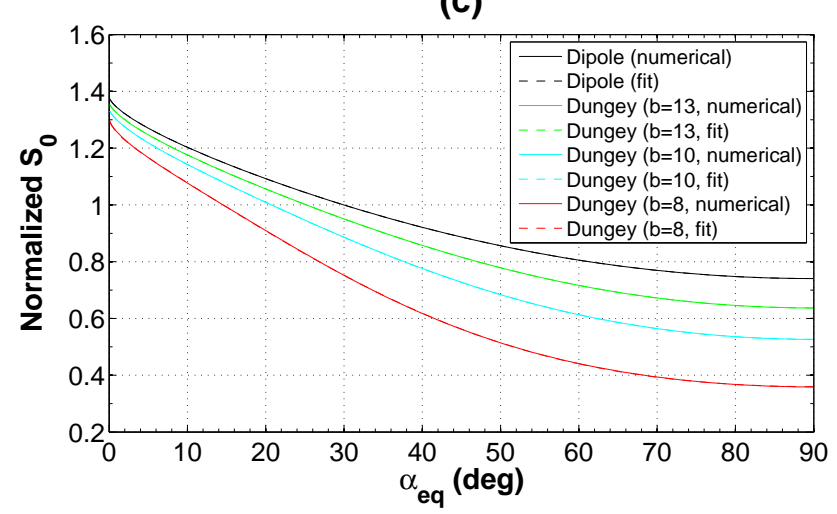

(b)

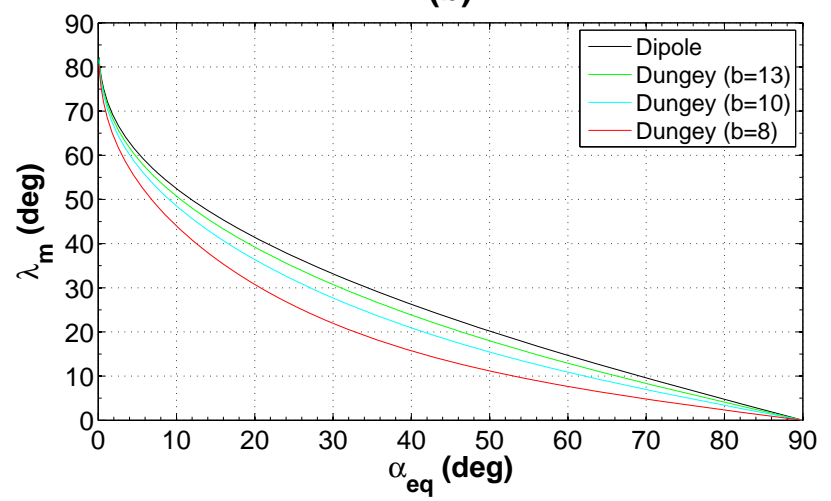

(d)

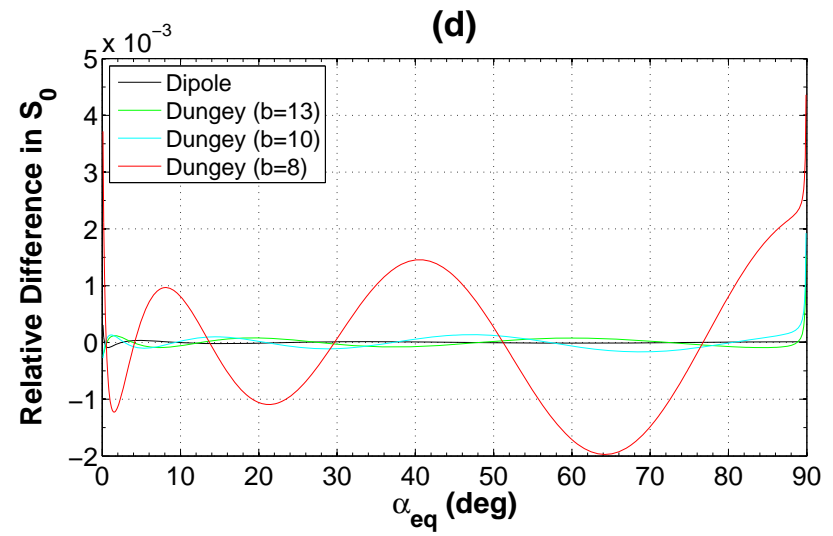

Fig. 2. (a) mirror radial distance $r_{\mathrm{m}}$ and (b) mirror latitude $\lambda \mathrm{m}$ as a function of $\alpha_{\mathrm{eq}}$ for $r_{0}=6 R_{\mathrm{E}}$ with $b / R_{\mathrm{E}}=\infty$ (dipole), 13,10 and 8 . (c) Solid curves: normalized bounce period related term $S_{0}$ calculated numerically using Eq. (31), dashes curves: approximate representations of $S_{0}$ by a fifth-order polynomial of $\sqrt{\sin \alpha_{\mathrm{eq}}}$, and (d) the errors associated with the fitted polynomials, for the indicated four cases of $b$-value.

\subsection{Bounce-averaged quasi-linear diffusion coefficients $\left\langle D_{\alpha \alpha}\right\rangle,\left\langle D_{\alpha p}\right\rangle / p$ and $\left\langle D_{p p}\right\rangle / p^{2}$}

Changes in ambient magnetic field introduce changes in resonant interactions of charged particles with any plasma wave mode and consequently alter wave-driven scattering effects on the particles. In this study we focus on resonances between electrons and whistler-mode chorus waves, since these emissions play an important dual role in both the loss and acceleration of radiation belt energetic electrons (Bortnik and Thorne, 2007) and act as the dominant contributor to the occurrence of nightside diffuse aurora in the inner magnetosphere (e.g., Thorne et al., 2010; Ni et al., 2011a, b). Following Glauert and Horne (2005) and Horne et al. (2005a), we have assumed a Gaussian spread of nightside chorus wave power spectral density with peak frequency $f_{\mathrm{m}} / f_{\mathrm{ce}}=$ 0.35 , lower cutoff frequency $f_{\mathrm{lc}} / f_{\mathrm{ce}}=0.05$, upper cutoff frequency $f_{\mathrm{uc}} / f_{\mathrm{ce}}=0.65$, and frequency width $\Delta f / f_{\mathrm{ce}}=0.35$, where $f_{\mathrm{ce}}$ is the equatorial electron gyrofrequency. The wave normal angle distribution is also assumed to be Gaussian, confined to the angular interval from $0^{\circ}$ to $45^{\circ}$ with peak wave normal angle as $0^{\circ}$ and angular width as $30^{\circ}$. We further assume that nightside chorus waves are confined to within $15^{\circ}$ of the magnetic equator with constant wave amplitude of $50 \mathrm{pT}$ along the magnetic field line. A constant cold electron $\left(N_{0}\right)$ density model with magnetic latitude is adopted along with the statistical trough plasma density model from Sheeley et al. (2001), i.e., $N_{0}=4.96 \mathrm{~cm}^{-3}$ for the case of $r_{0}=6 R_{\mathrm{E}}$ and $N_{0}=0.79 \mathrm{~cm}^{-3}$ for the case of $r_{0}=9 R_{\mathrm{E}}$. Our calculations include contributions from the $N=-5$ to $N=5$ cyclotron harmonic resonances and the Landau resonance $N=0$.

Figure 4 presents the bounce-averaged diffusion coefficients (from top to bottom: $\left\langle D_{\alpha \alpha}\right\rangle,\left\langle D_{p p}\right\rangle / p^{2}$ and $\left\langle D_{\alpha p}\right\rangle / p$ ) as a function of equatorial pitch angle $\alpha_{\mathrm{eq}}$ and electron kinetic energy $E_{k}$ at $r_{0}=6 R_{\mathrm{E}}$ for four Dungey magnetic fields (from left to right: $b / R_{\mathrm{E}}=\infty$ (dipole), 13, 10, and 8). There are a number of important features to address regarding chorus driven resonant scattering in the Dungey magnetic fields: (1) As $b$ decreases (the southward IMF increases), resonant scattering rates cover a broader range of electron energy and extend to equatorial pitch angles closer to $90^{\circ}$. The shift of diffusion to energies well below $\sim 1 \mathrm{keV}$ is mainly due to the decrease in minimum resonant electron energy associated with the decrease in equatorial magnetic field strength. The extension to higher $\alpha_{\text {eq }}$ can be a combined product of 
(a)

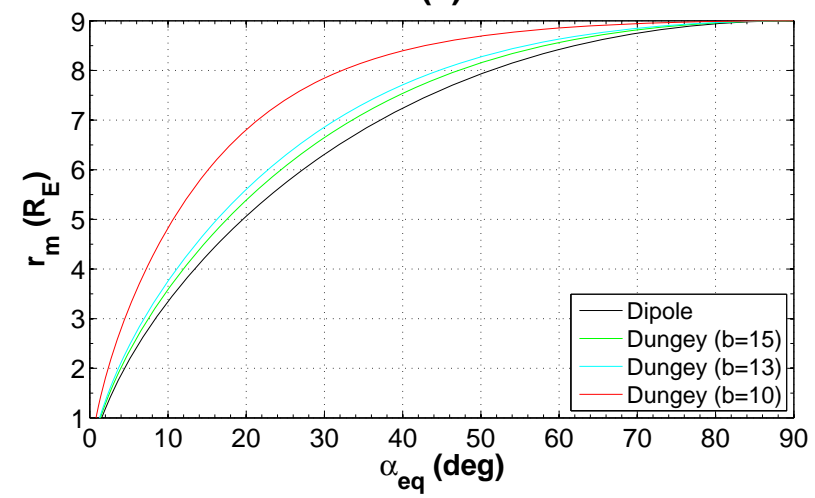

(c)

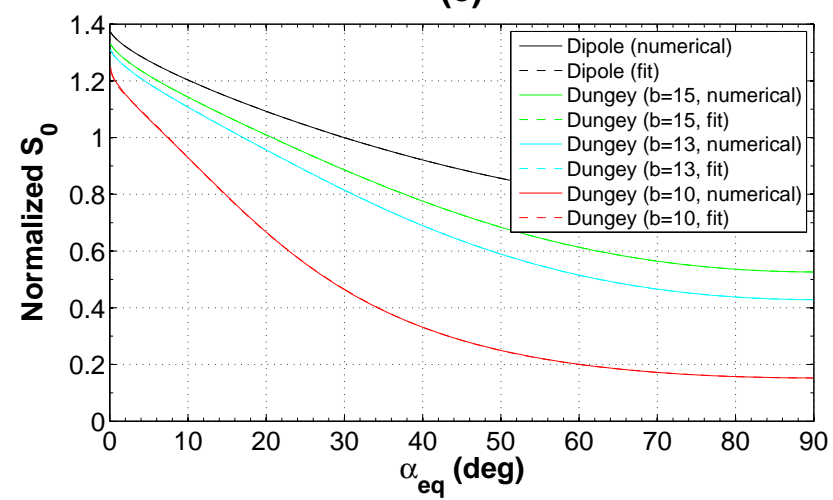

(b)

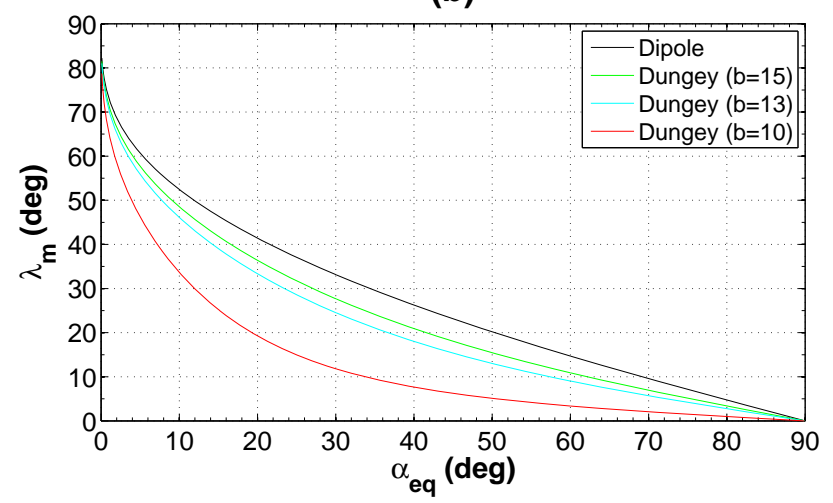

(d)

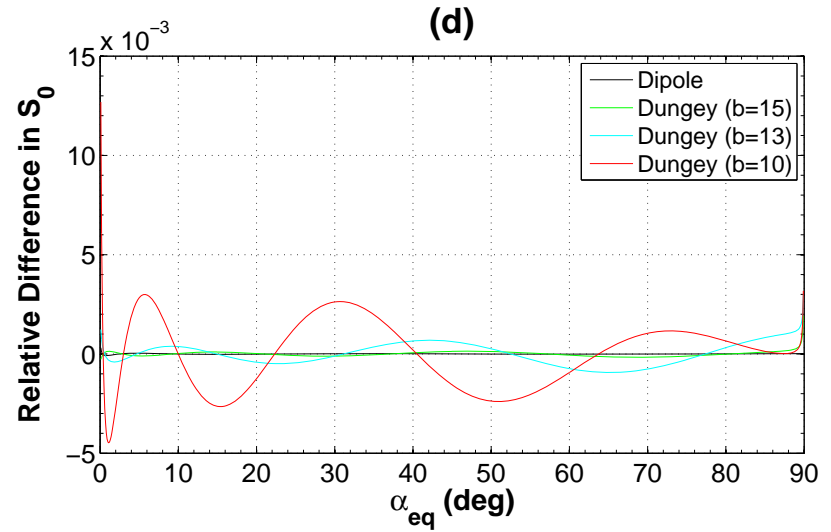

Fig. 3. Same as Fig. 2, except for $r_{0}=9 R_{\mathrm{E}}$ with $b / R_{\mathrm{E}}=\infty$ (dipole), 15, 13 and 10.

Table 1. Fitted values of six polynomial coefficients $\left(a_{0}-a_{5}\right)$ for approximate representations of normalized bounce period related term $S_{0}$ as a fifth-order polynomial of $\sqrt{\sin \alpha_{\mathrm{eq}}}$, corresponding to the indicated multiple choices of $b / R_{\mathrm{E}}$ at the equatorial crossings of $r_{0}=6 R_{\mathrm{E}}$ and $r_{0}=9 R_{\mathrm{E}}$ in the Dungey magnetosphere.

\begin{tabular}{ccccccc}
\hline \multicolumn{7}{c}{ Equatorial crossing $r_{0}=6 R_{\mathrm{E}}$} \\
\hline$b / R_{\mathrm{E}}$ & $a_{5}$ & $a_{4}$ & $a_{3}$ & $a_{2}$ & $a_{1}$ & $a_{0}$ \\
\hline$\infty$ & 0.2596 & -0.6787 & 0.6981 & -0.7017 & -0.22 & 1.3831 \\
13 & 0.7312 & -1.8302 & 1.5864 & -1.0304 & -0.182 & 1.3619 \\
10 & 1.339 & -3.0432 & 2.314 & -1.2492 & -0.1734 & 1.3392 \\
8 & 1.019 & -1.0903 & -0.4249 & -0.0216 & -0.4372 & 1.3146 \\
\hline \multicolumn{7}{c}{ Equatorial crossing $r_{0}=9 R_{\mathrm{E}}$} \\
\hline$b / R_{\mathrm{E}}$ & $a_{5}$ & $a_{4}$ & $a_{3}$ & $a_{2}$ & $a_{1}$ & $a_{0}$ \\
\hline$\infty$ & 0.2596 & -0.6787 & 0.6981 & -0.7017 & -0.22 & 1.3831 \\
15 & 1.339 & -3.0432 & 2.314 & -1.2492 & -0.1734 & 1.3392 \\
13 & 1.484 & -2.8362 & 1.6054 & -0.8781 & -0.2685 & 1.3226 \\
10 & -5.6968 & 16.9537 & -16.4604 & 5.3769 & -1.3167 & 1.2959 \\
\hline
\end{tabular}

variations in geomagnetic field and wave dispersion relation; (2) as $b$ decreases, the peak of $\left\langle D_{\alpha \alpha}\right\rangle$ at small $\alpha_{\text {eq }}$ tends to occur at lower energies, varying from $\sim 10 \mathrm{keV}$ in the dipolar field to $\sim 3 \mathrm{keV}$ when $b / R_{\mathrm{E}}=8$; pitch angle scattering at intermediate and high $\alpha_{\mathrm{eq}}$ also becomes more intense for 10 's $-100 \mathrm{keV}$ electrons; (3) when the southward IMF is en- hanced, momentum diffusion peaks at lower electron energies, shifting from $\sim 3 \mathrm{keV}$ in the dipolar field to $\sim 1 \mathrm{keV}$ in the greatly compressed Dungey magnetosphere; (4) The rates of mixed diffusion vary with $b$-value, following a trend similar to that for pitch angle scattering rates and momentum diffusion rates. 

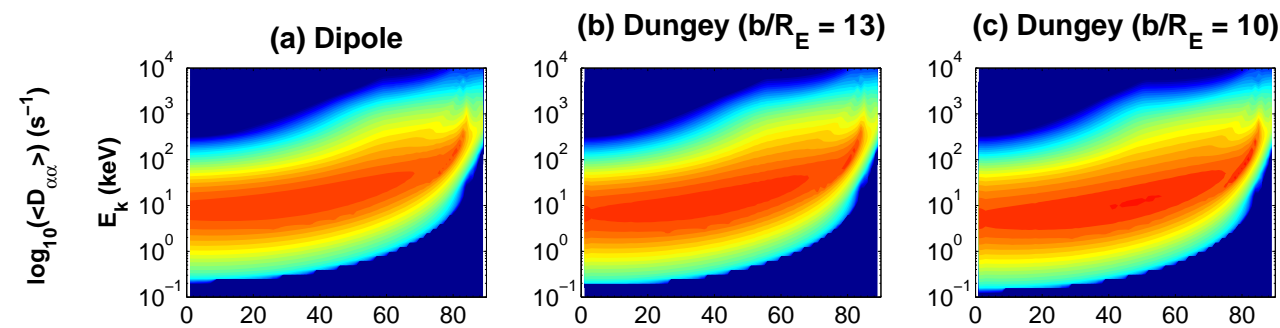

(d) Dungey $\left(b / R_{E}=8\right)$
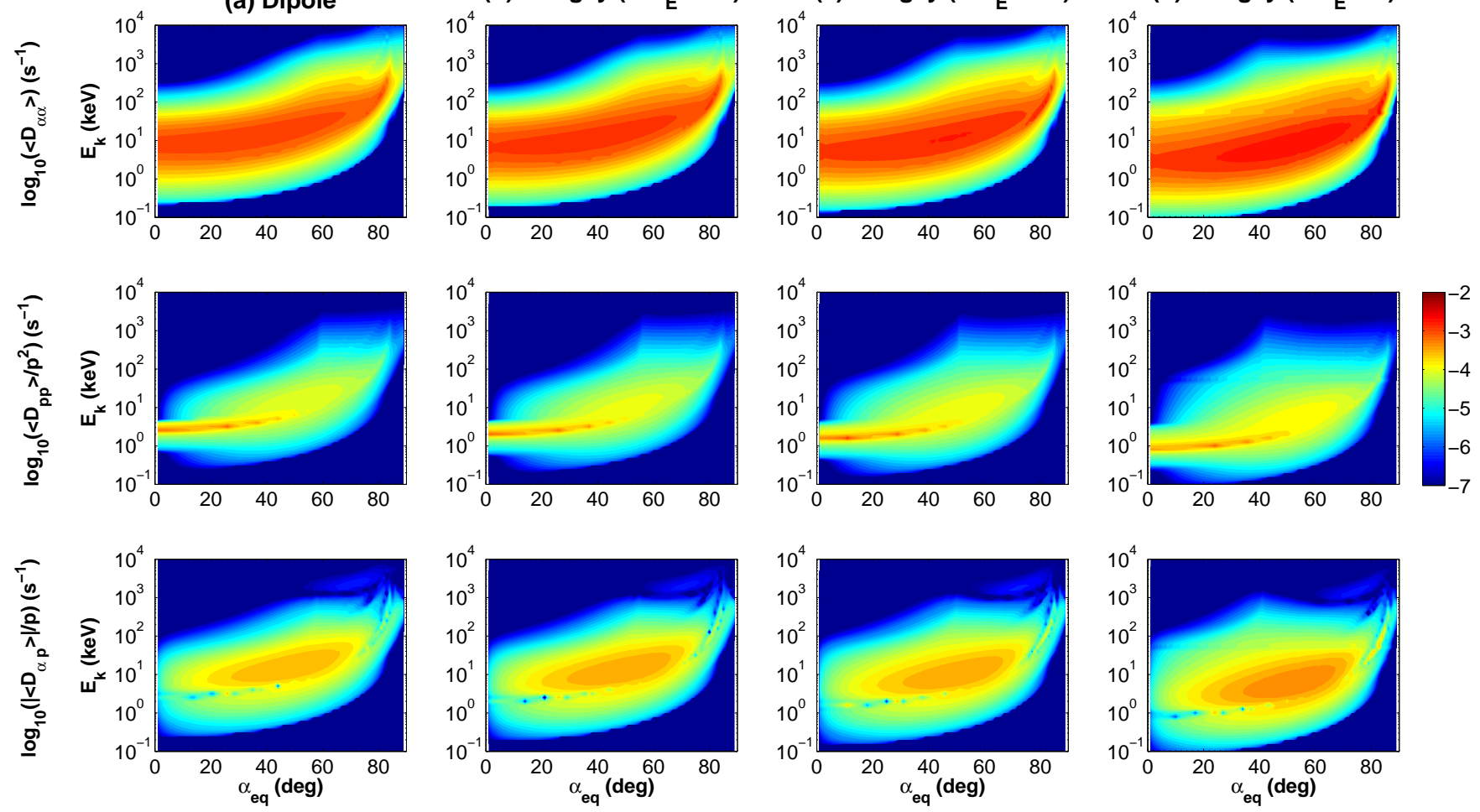

Fig. 4. Bounce-averaged diffusion rates (from top to bottom: $\left\langle D_{\alpha \alpha}\right\rangle,\left\langle D_{p p}\right\rangle / p^{2}$ and $\left\langle D_{\alpha p}\right\rangle / p$ ) as a function of equatorial pitch angle $\alpha_{\mathrm{eq}}$ and electron energy $E_{k}$ at $r_{0}=6 R_{\mathrm{E}}$ for the four Dungey magnetic fields (from left to right: $b / R_{\mathrm{E}}=\infty$ (dipole), 13, 10, and 8).
(a) $316 \mathrm{eV}$
(b) $1 \mathrm{keV}$
(c) $3.16 \mathrm{keV}$
(d) $10 \mathrm{keV}$
$\begin{array}{lll}\text { (e) } 31.6 \mathrm{keV} & \text { (f) } 100 \mathrm{keV}\end{array}$
(g) $316 \mathrm{keV}$
(h) $1 \mathrm{MeV}$
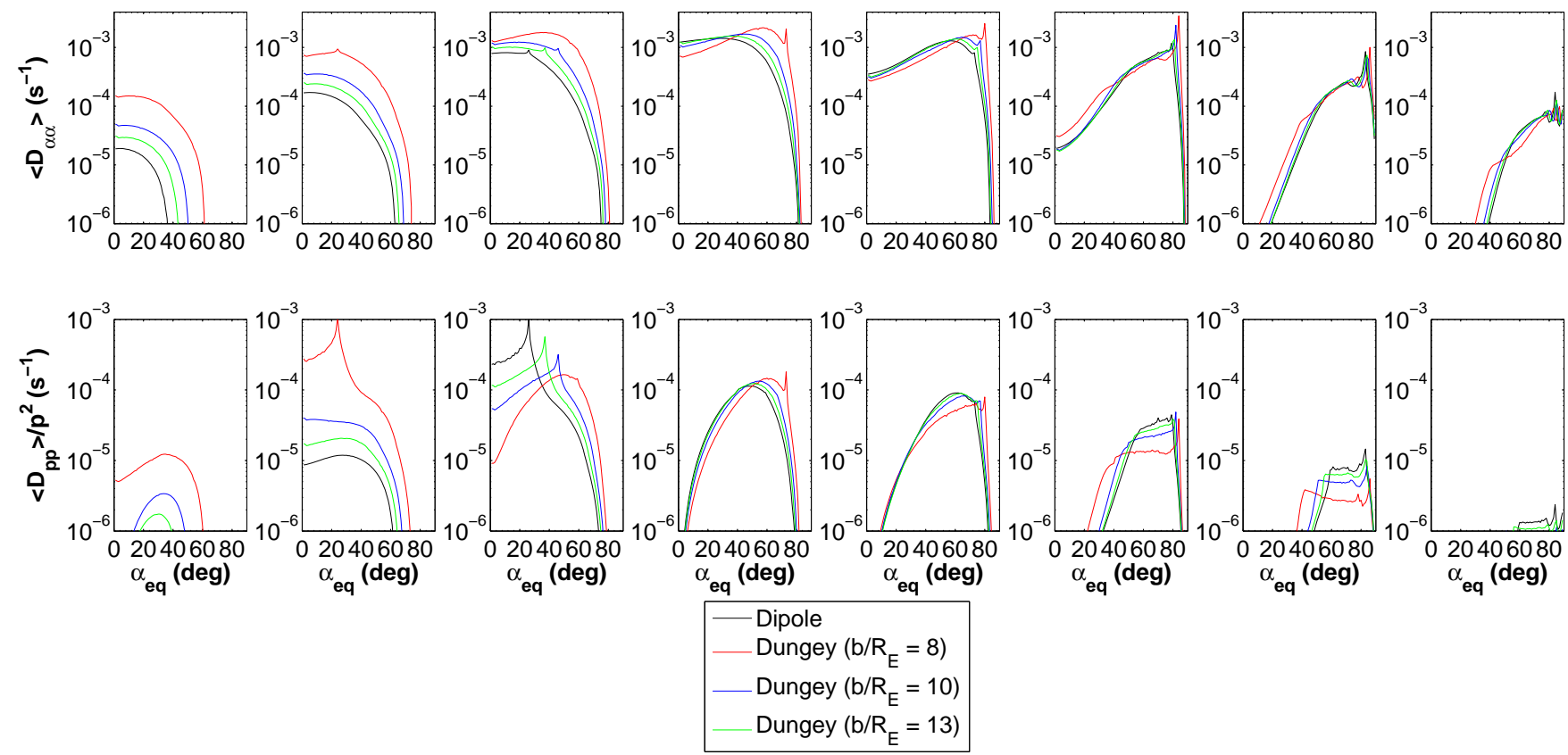

Fig. 5. $\left\langle D_{\alpha \alpha}\right\rangle$ and $\left\langle D_{p p}\right\rangle / p^{2}$ as a function of equatorial pitch angle $\alpha_{\mathrm{eq}}$ in the four Dungey magnetic fields at $r_{0}=6 R_{\mathrm{E}}$ for eight specific energies from $316 \mathrm{eV}$ to $1 \mathrm{MeV}$. 

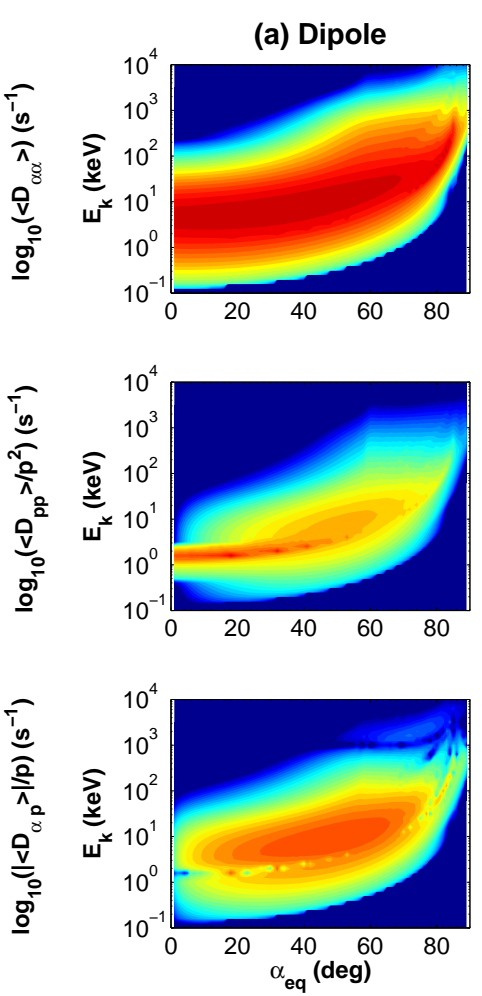

(b) Dungey $\left(b / R_{E}=15\right)$
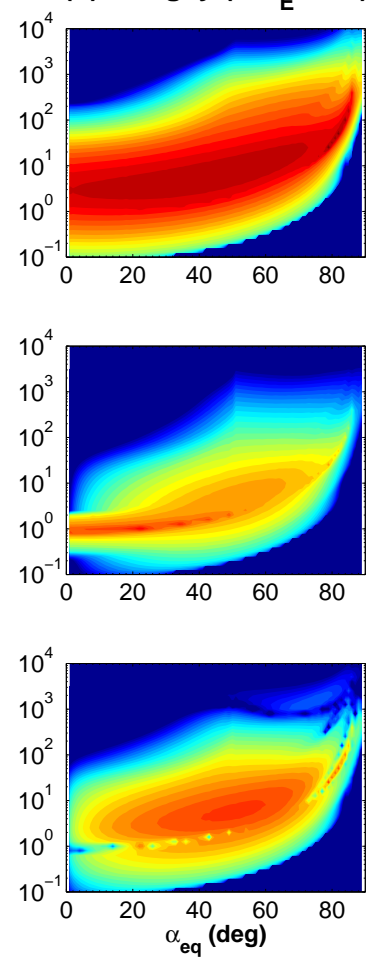

(c) Dungey $\left(b / R_{E}=13\right)$

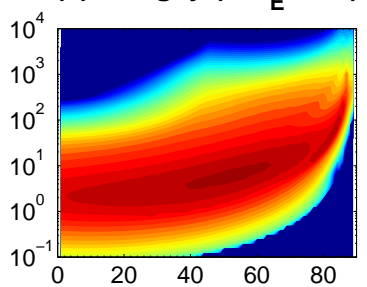

(d) Dungey $\left(b / R_{E}=10\right)$
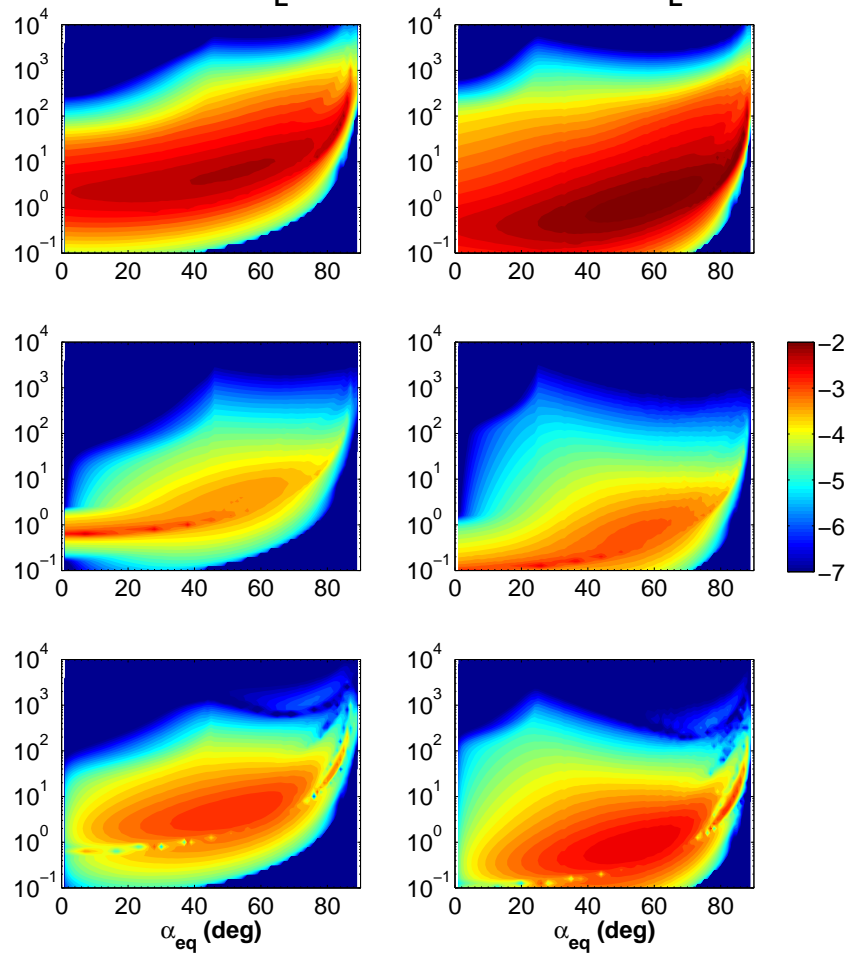

Fig. 6. Same as Fig. 4 , expect for $r_{0}=9 R_{\mathrm{E}}$ with $b / R_{\mathrm{E}}=\infty$ (dipole), 15,13 and 10.

Correspondingly, Fig. 5 shows the bounce-averaged rates of pitch angle scattering and momentum diffusion in the Dungey magnetic fields at eight specific energies ranging from $316 \mathrm{eV}$ to $1 \mathrm{MeV}$ that cover both plasma sheet source electrons and radiation belt relativistic electrons. The differences in scattering rates introduced by use of different Dungey field models depend strongly on electron energy. By comparing the results of $b / R_{\mathrm{E}}=\infty$ (dipole) and $b / R_{\mathrm{E}}=8$, which constantly exhibit the largest difference with each other, we can see that largest increase in diffusion rates, by a factor of well above 5 , occurs for $316 \mathrm{eV}$ and $1 \mathrm{keV}$ regardless of $\alpha_{\text {eq }}$. For $3.16 \mathrm{keV}$ electrons, $\left\langle D_{\alpha \alpha}\right\rangle$ increases but $\left\langle D_{p p}\right\rangle / p^{2}$ decreases as $b$ decreases. In contrast, $\left\langle D_{\alpha \alpha}\right\rangle$ decreases slightly at low and intermediate pitch angles for $10 \mathrm{keV}$ and $31.6 \mathrm{keV}$ electrons when the southward IMF is stronger. For $100 \mathrm{keV}, 316 \mathrm{keV}$, and $1 \mathrm{MeV}$ electrons, both pitch angle scattering and momentum diffusion rates increase with decreasing $b$-value at $\alpha_{\text {eq }}<\sim 50^{\circ}$. To summarize, the compression of Dungey field can produce scattering loss of plasma sheet electrons $<\sim 4 \mathrm{keV}$ and radiation belt electrons $>\sim 100 \mathrm{keV}$ on a timescale shorter than that in a dipolar field and induce momentum diffusion at high $\alpha_{\mathrm{eq}}$ closer to $90^{\circ}$.

For the Dungey magnetic field case of $r_{0}=9 R_{\mathrm{E}}$ with $b / R_{\mathrm{E}}=\infty$ (dipole), 15, 13, and 10, we show the 2-D plots of bounce-averaged diffusion coefficients in Fig. 6 and the line plots of $\left\langle D_{\alpha \alpha}\right\rangle$ and $\left\langle D_{p p}\right\rangle / p^{2}$ for the same eight energies in Fig. 7. We capture the similarities that resonant scat- tering rates cover a broader range of electron energy and equatorial pitch angle and increase considerably when the Dungey magnetosphere becomes more compressed. On the other hand, there are striking features distinct from the results for $r_{0}=6 R_{\mathrm{E}}$ : (1) The resonant diffusion can occur at much lower electron energies, say, $<\sim 100 \mathrm{eV}$, and can extend to equatorial pitch angles very close to $90^{\circ}$; (2) use of different Dungey magnetic fields tend to introduce larger variations in diffusion rates with the peaks of scattering rates shifting to lower energies, i.e., $\sim$ a few $\mathrm{keV}$ for $\left\langle D_{\alpha \alpha}\right\rangle$, and well below $1 \mathrm{keV}$ for $\left\langle D_{p p}\right\rangle / p^{2}$; (3) enhanced scattering loss occurs only for plasma sheet electrons with $E_{k}<\sim 1 \mathrm{keV}$. For radiation belt energetic electrons, more pronounced increases in $\left\langle D_{\alpha \alpha}\right\rangle$ and $\left\langle D_{p p}\right\rangle / p^{2}$ are present for $100 \mathrm{keV}$ to $1 \mathrm{MeV}$ when $b / R_{\mathrm{E}}=10$. The intensification of pitch angle scattering of $1 \mathrm{MeV}$ electrons in the compressed Dungey magnetic field at higher radial distances is consistent with the results of Orlova and Shprits (2010) showing that the differences in $\left\langle D_{\alpha \alpha}\right\rangle$ between use of the dipolar field and the Tsyganenko 89 model parameterized by Kp index are largest at the magnetic field line with the highest equatorial crossing.

As pointed out by Orlova and Shprits (2010) and Ni et al. (2011c), changes in magnetic field strength along the field line alter the wave dispersion relation and the waveparticle resonance condition so that electrons can resonate with the waves for the same equatorial pitch angle at lower latitudes on the nightside for the non-dipolar field than in 
$\begin{array}{lllllll}\text { (a) } 316 \mathrm{eV} & \text { (b) } 1 \mathrm{keV} & \text { (c) } 3.16 \mathrm{keV} & \text { (d) } 10 \mathrm{keV} & \text { (e) } 31.6 \mathrm{keV} & \text { (f) } 100 \mathrm{keV} & \text { (g) } 316 \mathrm{keV}\end{array}$

(h) $1 \mathrm{MeV}$
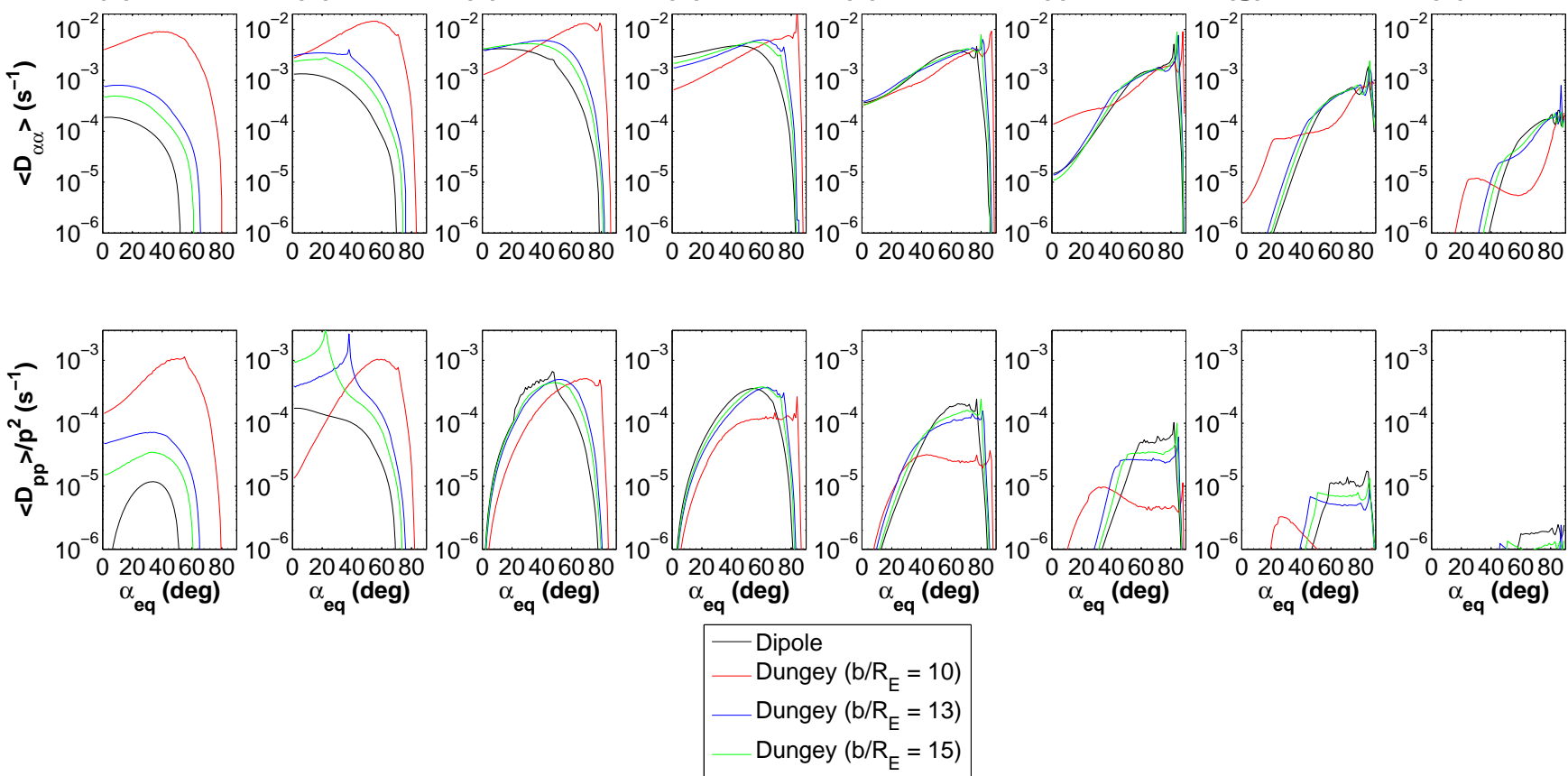

Fig. 7. Same as Fig. 5, expect for $r_{0}=9 R_{\mathrm{E}}$ with $b / R_{\mathrm{E}}=\infty$ (dipole), 15, 13 and 10.

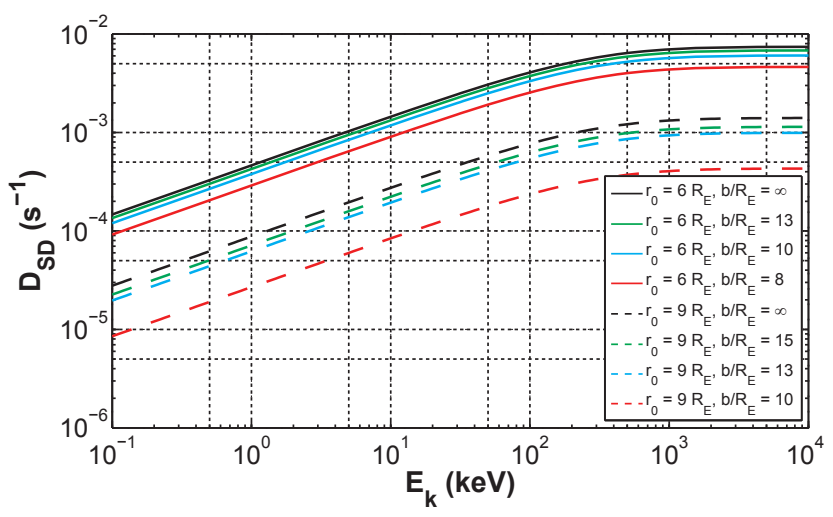

Fig. 8. Plots of strong diffusion rate $D_{\mathrm{SD}}$ as a function of electron energy $E_{k}$ for the two specific Dungey magnetic field lines with the indicated multiple $b$-values.

the dipolar field. Decrease in magnetic field strength near the equatorial region in the non-dipolar models lowers the minimum resonant energy of electrons interacting with chorus waves, which can explain the pronounced increase in scattering rates of 100 's $\mathrm{eV}$ electrons when $b$ decreases, i.e., the ambient magnetic field becomes more compressed. In addition, changes in the field line configuration can lead to changes in the electron bounce period, as shown in Sect. 3.2, which also contributes to the differences in bounce-averaged diffusion coefficients using non-dipolar fields.

\subsection{Strong diffusion rate $D_{\text {SD }}$}

By describing an extreme situation that particles can be scattered across the loss cone in times comparable to or less than the bounce period, strong diffusion is an essential concept of resonant wave-particle interactions. Under the limit of strong diffusion, particles diffuse across the loss cone within a quarter-bounce period, and the particle flux inside the loss cone approaches isotropy. Moreover, the particle precipitation rate is then insensitive to the magnitude of diffusion coefficients. Thus, quantitative comparison of quasi-linear diffusion coefficients with the strong diffusion rate can help us identify the efficiency of wave-induced scattering effects and evaluate the temporal evolution of particle pitch angle distribution and resultant precipitation loss. For example, Chen and Schulz (2001a, b) found numerically that scattering below strong diffusion is required to better model the observed precipitating electron energy fluxes near dawn and in the morning quadrant. Ni et al. (2011d) also estimated the diffuse auroral precipitation fluxes based upon the energy dependent loss cone filling index computed as the ratio between $\left\langle D_{\alpha \alpha}\right\rangle$ at the edge of equatorial loss cone and strong diffusion rate $D_{\mathrm{SD}}$. Following Kennel (1969), the definitive formula of strong diffusion rate is

$D_{\mathrm{SD}}=2\left(\alpha_{\mathrm{LC}}\right)^{2} / \tau_{\mathrm{B}}$,

where $\tau_{\mathrm{B}}$ is the bounce period given by Eq. (8), and $\alpha_{\mathrm{LC}}$ is the equatorial loss cone angle, which can be determined by $\alpha_{\mathrm{LC}}=\sin ^{-1}\left(B_{\mathrm{eq}} / B_{\mathrm{f}}\right)$ with $B_{\mathrm{f}}$ as the magnetic field intensity 
at the footprint latitude $\lambda_{\mathrm{f}}$. Apparently, both $\tau_{\mathrm{B}}$ and $\alpha_{\mathrm{LC}}$ depend on the adoption of magnetic field model, therefore strong diffusion rate $D_{\mathrm{SD}}$ varies with respect to magnetic field model. For the Dungey magnetic field models adopted in this study, it is easy to evaluate $\alpha_{\mathrm{LC}}$ according to the latitudinal distribution of Dungey magnetic field strength. We obtain that at $r_{0}=6 R_{\mathrm{E}} \alpha_{\mathrm{LC}}=2.85^{\circ}, 2.71^{\circ}, 2.53^{\circ}$, and $2.18^{\circ}$ for $b / R_{\mathrm{E}}=\infty$ (dipole), 13,10, and 8 , respectively and at $r_{0}=9 R_{\mathrm{E}} \alpha_{\mathrm{LC}}=1.53^{\circ}, 1.36^{\circ}, 1.26^{\circ}$, and $0.8^{\circ}$ for $b / R_{\mathrm{E}}=\infty$ (dipole), 15, 13, and 10, respectively. Evidently, the equatorial loss cone turns smaller due to the tendency of decrease in geomagnetic field amplitude in the equatorial region and increase at high latitudes. As the southward IMF intensifies or the $b$-value decreases, $\alpha_{\mathrm{LC}}$ decreases more markedly compared to the dipolar results. With the values of $\alpha_{\mathrm{LC}}$ available, we can compute the corresponding energy independent bounce period related term $S_{0}$ (normalized to $r_{0}$ ) as the following: at $r_{0}=6 R_{\mathrm{E}}, S_{0}=1.3053,1.2861,1.2636$, and 1.2253 for $b / R_{\mathrm{E}}=\infty$ (dipole), 13,10 , and 8 , respectively; at $r_{0}=9 R_{\mathrm{E}}, S_{0}=1.3310,1.2895,1.268$, and 1.1959 for $b / R_{\mathrm{E}}=\infty$ (dipole), 15,13 , and 10, respectively. Clearly, $S_{0}$ (or $\tau_{B}$ ) decreases with decreasing $b$, due to enhanced compression of the Dungey magnetosphere.

In Fig. 7 we show plots of $D_{\mathrm{SD}}$ as a function of electron energy $E_{k}$ for the two specific Dungey magnetic field lines with the indicated $b$-values. $D_{\mathrm{SD}}$ is a monotonically increasing function of $E_{k}$ for below $1 \mathrm{MeV}$ electrons and keeps almost unchanged for energies exceeding $1 \mathrm{MeV}$, in agreement with the results of Summers and Thorne (2003). When the Dungey magnetic field line moves inward from $r_{0}=9 R_{\mathrm{E}}$ to $r_{0}=6 R_{\mathrm{E}}, D_{\mathrm{SD}}$ decreases substantially by about an order of magnitude at all energies. In addition, $D_{\mathrm{SD}}$ also decreases considerably when the compression of the Dungey magnetosphere is enhanced by intensified southward IMF, and the degree of decrease in $D_{\mathrm{SD}}$ is larger for the magnetic field line with higher equatorial crossing $r_{0}$. The feature that strong diffusion limit occurs at a lower diffusion rate under disturbed geomagnetic conditions is important, since it suggests that during periods of magnetic storms/substorms magnetospheric electrons can find it easier to expose themselves to wave-induced intense scattering at a rate reaching or comparable to $D_{\mathrm{SD}}$ and therefore undergo enhanced precipitation loss near the loss cone.

\section{Discussions}

While the bounce-averaged Fokker-Planck diffusion equation has been studied extensively in a dipolar geomagnetic field, in this study we have extended it to a generalized form (Eq. 22) applicable to a geometry equivalent to a flux tube of essentially arbitrary shape that assumes only that it yields two mirror points for each trapped particle. Without recourse to a dipolar field assumption, we have obtained the similar form of bounce-averaged diffusion equation regardless of de- tails of the mirror geometry. We have adopted a straightforward method through integration averaging over the bounce period to perform the bounce-averaging of localized FokkerPlanck equation, and we notice that Schulz (1976) carried out the bounce-averaging procedure in geometric mirrors by calculating the Jacobian determinant. The bounce-averaged pitch angle diffusion equation that Schulz (1976) obtained in the limit of weak diffusion is consistent with the term associated with pure pitch angle diffusion in Eq. (22), but our equation gives a full description of bounce-averaged FokkerPlanck diffusion equation in arbitrary 2-D magnetic field including pitch angle scattering, momentum diffusion, and mixed terms.

Although the bounce-averaged Fokker-Planck diffusion equation at a constant Roederer $L$ retains a similar expression for arbitrary magnetic field, non-negligible or even substantial changes in bounce period and bounce-averaged diffusion coefficients with respect to magnetic field model demonstrate the importance of the use of realistic magnetic field representations to quantifying resonant wave-particle interactions and resultant contributions to particle dynamics. For purposes of quantitative comparisons with the commonly used dipolar field, we have adopted multiple Dungey magnetic fields parameterized by the intensity of southward IMF. The Dungey models take into account the compression of magnetic field resulting from solar wind disturbances, thus a better approximation than a dipole to the ambient magnetic field, however, they cannot deal with the tilting or twisting of magnetic field lines. Tsyganenko model series, using the solar wind parameters and geomagnetic activity indexes as input, can model the geomagnetic fields under various geomagnetic conditions with better accuracy. Orlova and Shprits (2010) and Ni et al. (2011c) have used the Tsyganenko models to compute bounce-averaged diffusion rates for $1 \mathrm{MeV}$ relativistic electrons and diffuse auroral electrons, respectively. They found that the scattering rates are strongly dependent on the adoption of magnetic field model and that most accurate evaluation of wave induced particle diffusion effects requires most reliable representations of background magnetic field. Note that since the Tsyganenko magnetic fields are usually tilted, i.e., non-axisymmetric with minimum geomagnetic field present at non-zero degree of magnetic latitude, a half-bounce integral instead of a quarter-bounce integral should be implemented for calculation of $S_{0}$ and bounceaveraged diffusion coefficients.

To evaluate the bounce-averaged diffusion coefficients which are key parameters for numerically solving the Fokker-Planck diffusion equation, we also adopted an empirical single-band model of chorus waves without the gap at a half electron gyrofrequency, following previous studies. Statistical analyses of CRRES wave datasets (Meredith et al., 2001, 2009b; Ni et al., 2011b) have captured a clear dual-band structure of chorus emissions and established an improved model of lower band and upper band chorus as a function of L-shell, magnetic local time (MLT), and level of 
geomagnetic activity. Since knowledge of wave power distribution is critical to the quantification of resonant scattering rates (Shprits et al., 2006b; Summers and Ni, 2008), the companion paper by Ma et al. (2012) uses the more accurate chorus wave information obtained based upon CRRES wave observations to compute the matrices of bounce-averaged diffusion coefficients in 2-D Dungey magnetic fields, which are subsequently taken as inputs to solve the bounce-averaged Fokker-Planck diffusion equation for modeling the temporal evolution of plasma sheet electron pitch angle distribution and exploring the effects of non-dipolar magnetic fields. We have made an approximate comparison of the magnitude of diffusion coefficients between using a dipolar field with improved nightside chorus (lower-band and upper-band) wave information (Fig. 6 of Ni et al., 2011b) and using a Dungey field with less accurate wave information (Fig. 5 in the present study). For $1 \mathrm{keV}$ electrons, there is a decrease, by a factor of $\sim 8$, in pitch angle scattering rates at lower equatorial pitch angles when we use the Dungey magnetic field but less accurate wave information. In contrast, for $10 \mathrm{keV}$ electrons, the magnitude of decrease in scattering rates becomes smaller, within a factor of $\sim 3$. This suggests that quantitative evaluation of the relative importance of using a non-dipolar magnetic field with crude wave model or using a dipole magnetic field with improved wave model is sensitive to a number of factors, such as electron energy, pitch angle, and also L-shell and geomagnetic condition, which needs a careful consideration. Overall, we can see that wave model can be most important to reasonably evaluate the magnitude of diffusion coefficients. In other words, descriptions of plasma waves with high accuracy are the first priority for us to comprehensively understand the processes of resonant wave-particle interactions. To fulfill it, long-term, largesize datasets from past, existing, and upcoming satellite missions are required and should be combined systematically for careful, detailed wave analyses, which is one outstanding subject of our ongoing studies. In contrast, consideration of non-dipolar magnetic field strength and configuration is of relatively lower priority. However, its effect is one important factor that should be taken into account to investigate resonant wave-particle interactions in the realistic magnetosphere, particularly during geomagnetically disturbed conditions when the ambient magnetic field is highly fluctuated and/or distorted.

To derive Eq. (22), we have assumed a constant Roederer $L$, equivalently, a uniform value of the third adiabatic invariant associated with particle drift motion. However, the particles of interest can have different "azimuthal" drift rates depending on equatorial pitch angle, so that they do not remain in the same flux tube with each other. Moreover, bounce period and bounce-averaged diffusion coefficients would also vary with azimuthal position (or with MLT) in most situations of interest, as would the size of the loss cone. For 2-D Fokker-Planck diffusion simulations, this raises a question of whether to perform a further average of the diffusion equation over azimuthal angle or (if not) whether to include some "advective" terms to account for the azimuthal drift of phase space density distribution that depends (at least near the loss cone) on the azimuthal coordinate. For example, Su et al. (2010b), by comparing the numerical results between MLT-dependent and MLT-averaged simulations, stated that azimuthal advection could play an important role in the radiation belt electron dynamics. Another more physically accurate option is to extend 2-D Fokker-Planck diffusion equation with respect to kinetic energy and equatorial pitch angle to 3-D or 4-D diffusion equation by adding one dimension of Roederer $L$ or two dimensions of Roederer $L$ and MLT. There are already a number of studies on this aspect (e.g., Varotsou et al., 2005, 2008; Fok et al., 2008; Jordanova et al., 2008, 2010; Shprits et al., 2009a, b, 2011; Albert et al., 2009; Xiao et al., 2010b; Su et al., 2010a, b; Subbotin et al., 2010, 2011), but further modeling efforts are required to develop a comprehensive and sophisticated global model of magnetospheric electron transport, acceleration, and loss in the realistic environment of ambient magnetic field under all levels of geomagnetic activity.

\section{Summary}

We have performed a detailed derivation of bounce-averaged Fokker-Planck diffusion equation applicable to arbitrary magnetic field at a constant Roederer $L$. The form of bounceaveraged diffusion equation is found regardless of details of the mirror geometry, which suggests that the numerical schemes developed for solving the modified 2-D FokkerPlanck equation in a magnetic dipole should be feasible for similar computation efforts on modeling wave-induced particle diffusion processes in any non-dipolar magnetic field. However, bounce period related term and bounce-averaged diffusion coefficients are required to be computed in realistic magnetic fields instead of using the commonly adopted dipolar model to pursue improved understanding of the dynamics of magnetospheric particles and the role of resonant wave-particle interactions.

With the application to the Dungey magnetic fields, which are axisymmetric with field line configuration and magnetic field strength controlled by the intensity of southward IMF, we have evaluated the bounce period related term, bounceaveraged scattering rates, and strong diffusion rate at two representative field lines with equatorial crossing of 6 and 9 Earth radii under different conditions of southward IMF. Regarding these key parameters for Fokker-Planck diffusion simulations, we find that

1. The normalized bounce period related term $S_{0}$ decreases when the southward IMF intensifies, mainly due to the shorter length of compressed field line. Compared to the results using the dipolar field, the degree of decrease in $S_{0}$ increases considerably with $\alpha_{\mathrm{eq}}$, and the extent of differences becomes larger for the field line with higher 
equatorial crossing. $S_{0}$ can be approximated with high accuracy by a fifth-order polynomial of $\sqrt{\sin \alpha_{\text {eq }}}$, which is useful to be incorporated into multi-dimensional diffusion simulations.

2. With enhanced southward IMF, bounce-averaged diffusion coefficients cover a broader range of $E_{k}$ and $\alpha_{\mathrm{eq}}$ (closer to $90^{\circ}$ ), and the rates tend to increase and peak at lower energies. In general, the compression of Dungey magnetosphere can produce scattering loss of plasma sheet electrons $<\sim 4 \mathrm{keV}$ and radiation belt electrons $>\sim 100 \mathrm{keV}$ on a timescale shorter than that in a dipolar field and induce momentum diffusion at high $\alpha_{\mathrm{eq}}$ closer to $90^{\circ}$, essentially resulting from the changes in wave dispersion relation and resonance condition introduced by changes in geomagnetic field distribution. Differences in scattering rates are also stronger for higher equatorial crossing.

3. Strong diffusion rate $D_{\mathrm{SD}}$ decreases considerably when the Dungey magnetosphere is more compressed, as a combined product of changes in both equatorial loss cone and bounce period. Decrease in $D_{\mathrm{SD}}$ for enhanced geomagnetic activity and higher equatorial crossing is important for understanding the behaviors of storm-time magnetospheric electrons and their global precipitation losses.

The advantages of this study can be mainly summarized as follows: (1) it confirms the need for adoption of realistic magnetic field representation to perform accurate determination of electron resonant scattering rates, and precise FokkerPlanck diffusion simulations of magnetospheric electron dynamics; (2) it establishes a reasonable fifth-order polynomial model (with high accuracy) of bounce period related term in non-dipolar Dungey magnetic fields, in contrast to the empirical approximations previously developed by Lencheck et al. (1961) (Eq. 19), Davidson (1976) (Eq. 6), and recently revisited by Orlova and Shprits (2011) (Eq. 15 and Table 1). This developed model can be readily incorporated into Fokker-Planck diffusion simulations using Dungey magnetic fields; (3) it computes the strong diffusion rates (an essential concept of resonant wave-particle interactions) corresponding to the Dungey magnetic fields for comparisons with the dipolar results, which has clearly indicated that changes in strong diffusion rate associated with changes in ambient magnetic field geometry can introduce considerable influences on the determination of the efficiency of wave-induced resonant scattering and thus affect the pitch angle distribution of electrons at different energies. The companion paper by Ma et al. (2012) will be a first attempt to utilize the derived general bounce-averaged Fokker-Planck diffusion equation and the reasonably fitted bounce period related terms to better understand the evolution of plasma sheet electron pitch angle distribution due to chorus induced scattering in a nondipolar geometry such as the Dungey magnetic fields.
The present study concentrates on using the Dungey magnetic field models, but it is obvious that, although better than the dipolar field, Dungey magnetic fields is still quite simple, compared to the more sophisticated Tsyganenko magnetic field model series and the recent event-adaptive magnetic field models of Kubyshkina et al. (2009, 2011), which are non-asixsymmetric, MLT and geomagnetic condition dependent by assuming physical mechanisms embodied by the solar wind inputs and contributions from different current systems. In addition, Kabin et al. (2007) have developed a modified, non-axisymmetric version of Dungey magnetic field to account for the MLT dependence of ambient magnetic field, with the stretching on the nightside and the compression on the dayside. All these more complicated magnetic field models with expected higher accuracy will be adopted in our future studies to pursue more precise evaluations of resonant wave-particle interactions. The simple model of nightside chorus waves based upon Glauert and Horne (2005) and Horne et al. (2005a) is another challenge to this study. We have noticed that there have been a number of recent studies of chorus wave characteristics based upon statistical analyses of satellite measurements including CLUSTER (Agapitov et al., 2011), POLAR (Haque et al., 2010; Bunch et al., 2011), CRRES (Ni et al., 2011b), and THEMIS (Li et al., 2009, 2011). We fully understand that a much better chorus wave model including reasonable wave normal angle distribution should be used to obtain more accurate scattering rates. Since this paper mainly aims to exhibit the effects of non-dipolar field on bounce-averaged Fokker-Planck diffusion equation and the companion paper (Ma et al., 2012) will adopt a greatly improved chorus wave model based upon $\mathrm{Ni}$ et al. (2011b) to perform Fokker-Planck diffusion simulations in Dungey magnetic fields, our present study has adopted a rather simple chorus wave model. Our further goal is to adopt the most reliable chorus wave model that we can approach based upon all the available satellite analyses to perform diffusion coefficient calculations and FokkerPlanck diffusion simulations using most accurate magnetic field models, which is outside the scope of this study but will be the subject of our following investigations. In addition, changes in cold plasma density can cause changes in wave induced scattering coefficients, mainly due to changes in wave dispersion relation and resonant frequencies (thus resonant wave power) for any specific electrons energy. In the present study we have adopted Sheeley et al. (2001) plasmatrough density model with constant latitudinal distribution. Use of more accurate cold plasma density model (especially taking into account the latitudinal variation, e.g., Denton et al., 2006) needs to be incorporated into our further studies. Finally, we note that a number of other electromagnetic wave modes, such as EMIC wave (e.g., Summers and Thorne, 2003; Summers, 2005; Summers et al., 2007b; Summers and Ni, 2008; Albert, 2008; Xiao et al., 2011b), plasmaspheric hiss (e.g., Lyons and Thorne, 1973; Meredith et al., 2006, 2007; Summers et al., 2008; Xiao et al., 2009a), 
magnetosonic waves (Horne et al., 2007), and superluminous waves (e.g., Xiao et al., 2006, 2010a, 2011a), also play more or less important roles in outer radiation belt dynamics. Use of non-dipolar magnetic fields will modify the wave dispersion relation and subsequently the resonant frequencies and resonant wave power of these waves in a manner similar to that for chorus waves. As a consequence, adoption of nondipolar magnetic fields will give rise to non-negligible and even important contribution to the quantification of resonant scattering coefficients induced by these waves, which will be investigated in detail in our following studies.

Acknowledgements. This research was supported in part by NSF grant ATM-0802843 and NASA grant NNX12AD12G. The authors would like to thank Michael Schulz and Yuri Shprits for useful discussions. We also thank the reviewers for valuable and constructive comments on the manuscript.

Topical Editor R. Nakamura thanks two anonymous referees for their help in evaluating this paper.

\section{References}

Agapitov, O., Krasnoselskikh, V., Khotyaintsev, Y. V., and Rolland, G.: A statistical study of the propagation characteristics of whistler waves observed by Cluster, Geophys. Res. Lett., 38, L20103, doi:10.1029/2011GL049597, 2011.

Albert, J. M.: Using quasi-linear diffusion to model acceleration and loss from wave-particle interactions, Space Weather, 2, S09S03, doi:10.1029/2004SW000069, 2004.

Albert, J. M.: Evaluation of quasi-linear diffusion coefficients for whistler mode waves in a plasma with arbitrary density ratio, J. Geophys. Res., 110, A03218, doi:10.1029/2004JA010844, 2005.

Albert, J. M.: Efficient approximations of quasi-linear diffusion coefficients in the radiation belts, J. Geophys. Res., 113, A06208, doi:10.1029/2007JA012936, 2008.

Albert, J. M.: Diffusion by one wave and by many waves, J. Geophys. Res., 115, A00F05, doi:10.1029/2009JA014732, 2010.

Albert, J. M., Meredith, N. P., and Horne, R. B.: Three-dimensional diffusion simulation of outer radiation belt electrons during the 9 October 1990 magnetic storm, J. Geophys. Res., 114, A09214, doi:10.1029/2009JA014336, 2009.

Beutier, T. and Boscher, D.: A three-dimensional analysis of the electron radiation belt by the Salammbô code, J. Geophys. Res., 100, 14853-14861, 1995.

Bortnik, J. and Thorne, R. M.: The dual role of ELF/VLF chorus waves in the acceleration and precipitation of radiation belt electrons, J. Atmos. Solar-Terr. Phys., 69, 378-386, 2007.

Bortnik, J. and Thorne, R. M.: Transit time scattering of energetic electrons due to equatorially confined magnetosonic waves, J. Geophys. Res., 115, A07213, doi:10.1029/2010JA015283, 2010.

Bortnik, J., Thorne, R. M., and Inan, U. S.: Nonlinear interaction of energetic electrons with large amplitude chorus, Geophys. Res. Lett., 35, L21102, doi:10.1029/2008GL035500, 2008.

Bourdarie, S., Boscher, D., Beutier, T., Sauvaud, J.-A., and Blanc, M.: Magnetic storm modeling in the Earth's electron belt by the Salammbô code, J. Geophys. Res., 101, 27171-27176, 1996.
Bunch, N. L., Spasojevic, M., and Shprits, Y. Y.: On the latitudinal extent of chorus emissions as observed by the Polar Plasma Wave Instrument, J. Geophys. Res., 116, A04204, doi:10.1029/2010JA016181, 2011.

Chen, M. and Schulz, M.: Simulations of storm time diffuse aurora with plasmasheet electrons in strong pitch angle diffusion, J. Geophys. Res., 106, 1873-1886, 2001a.

Chen, M., and Schulz, M.: Simulations of diffuse aurora with plasma sheet electrons in pitch angle diffusion less than everywhere strong, J. Geophys. Res., 106, 28949-28966, 2001 b.

Chen, M., Schulz, M., Lyons, L., and Gorney, D.: Stormtime transport of ring current and radiation belt ions, J. Geophys. Res., 98, 3835-3849, 1993.

Davidson, G.: An improved empirical description of the bounce motion of trapped particles, J. Geophys. Res., 81, 4029-4030, 1976.

Denton, R. E., Takahashi, K., Galkin, I. A., Nsumei, P. A., Huang, X., Reinisch, B. W., Anderson, R. R., Sleeper, M. K., and Hughes, W. J.: Distribution of density along magnetospheric field lines, J. Geophys. Res., 111, A04213, doi:10.1029/2005JA011414, 2006.

Dungey, J. W.: Interplanetary magnetic field and the auroral zones, Phys. Rev. Letts., 6, 47-48, 1961.

Fok, M., Horne, R. B., Meredith, N. P., and Glauert, S. A.: Radiation Belt Environment model: Application to space weather nowcasting, J. Geophys. Res., 113, A03S08, doi:10.1029/2007JA012558, 2008.

Glauert, S. A. and Horne, R. B.: Calculation of pitch-angle and energy diffusion coefficients with the PADIE code, J. Geophys. Res., 110, A04206, doi:10.1029/2004JA010851, 2005.

Haque, N., Spasojevic, M., Santolík, O., and Inan, U. S.: Wave normal angles of magnetospheric chorus emissions observed on the Polar spacecraft, J. Geophys. Res., 115, A00F07, doi:10.1029/2009JA014717, 2010.

Horne, R. B. and Thorne, R. M.: Electron pitch angle diffusion by electrostatic electron cyclotron harmonic waves: The origin of pancake distributions, J. Geophys. Res., 105, 5391-5402, 2000.

Horne, R. B., Thorne, R. M., Meredith, N. P., and Anderson, R. R.: Diffuse auroral electron scattering by electron cyclotron harmonic and whistler mode waves during an isolated substorm, $\mathrm{J}$ Geophys. Res., 108, 1290, doi:10.1029/2002JA009736, 2003.

Horne, R. B., Thorne, R. M., Glauert, S. A., Albert, J. M., Meredith, N. P., and Anderson, R. R.: Timescale for radiation belt electron acceleration by whistler mode chorus waves, J. Geophys. Res., 110, A03225, doi:10.1029/2004JA010811, 2005a.

Horne, R. B., Thorne, R. M., Shprits, Y. Y., Meredith, N. P., Glauert, S. A., Smith, A. J., Kanekal, S. G., Baker, D. N., Engebretson, M. J., Posch, J. L., Spasojevic, M., Inan, U. S., Pickett, J. S., and Decreau, P. M.: Wave acceleration of electrons in the Van Allen radiation belts, Nature, 437, 227, doi:10.1038/nature03939, 2005 b.

Horne, R. B., Thorne, R. M., Glauert, S. A., Meredith, N. P., Pokhotelov, D., and Santolík, O.: Electron acceleration in the Van Allen radiation belts by fast magnetosonic waves, Geophys. Res. Lett., 34, L17107, doi:10.1029/2007GL030267, 2007.

Inan, U. S., Chiu, Y. T., and Davidson, G. T.: Whistler-mode chorus and morningside aurorae, Geophys. Res. Lett., 19, 653-656, 1992.

Johnstone, A., Walton, D., Liu, R., and Hardy, D.: Pitch angle diffusion of low-energy electrons by whistler mode waves, J. Geophys. Res., 98, 5959-5967, 1993. 
Jordanova, V. K., Albert, J., and Miyoshi, Y.: Relativistic electron precipitation by EMIC waves from self-consistent global simulations, J. Geophys. Res., 113, A00A10, doi:10.1029/2008JA013239, 2008.

Jordanova, V. K., Zaharia, S., and Welling, D. T.: Comparative study of ring current development using empirical, dipolar, and self-consistent magnetic field simulations, J. Geophys. Res., 115, A00J11, doi:10.1029/2010JA015671, 2010.

Kabin, K., Rankin, R., Mann, I. R., Degeling, A. W., and Marchand, R.: Polarization properties of standing shear Alfvén waves in non-axisymmetric background magnetic fields, Ann. Geophys., 25, 815-822, doi:10.5194/angeo-25-815-2007, 2007.

Kennel, C. F.: Consequences of a magnetospheric plasma, Rev. Geophys., 7, 379-419, 1969.

Kennel, C. F. and Engelmann, F.: Velocity space diffusion from weak plasma turbulence in a magnetic field, Phys. Fluids, 9, 2377-2388, 1966.

Kubyshkina, M., Sergeev, V., Tsyganenko, N., Angelopoulos, V., Runov, A., Singer, H., Glassmeier, K. H., Auster, H. U., and Baumjohann, W.: Toward adapted time-dependent magnetospheric models: A simple approach based on tuning the standard model, J. Geophys. Res., 114, A00C21, doi:10.1029/2008JA013547, 2009.

Kubyshkina, M., Sergeev, V., Tsyganenko, N., Angelopoulos, V., Runov, A., Donovan, E., Singer, H., Auster, U., and Baumjohann, W.: Time dependent magnetospheric configuration and breakup mapping during a substorm, J. Geophys. Res., 116, A00I27, doi:10.1029/2010JA015882, 2011.

Lenchek, A., Singer, S., and Wentworth, R.: Geomagnetically trapped electrons from cosmic ray albedo neutrons, J. Geophys. Res., 66, 4027-4046, 1961.

Li, W., Shprits, Y. Y., and Thorne, R. M.: Dynamic evolution of energetic outer zone electrons due to wave-particle interactions during storms, J. Geophys. Res., 112, A10220, doi:10.1029/2007JA012368, 2007.

Li, W., Thorne, R. M., Angelopoulos, V., Bortnik, J., Cully, C. M., Ni, B., LeContel, O., Roux, A., Auster, U., and Magnes, W.: Global distribution of whistler-mode chorus waves observed on the THEMIS spacecraft, Geophys. Res. Lett., 36, L09104, doi:10.1029/2009GL037595, 2009.

Li, W., Bortnik, J., Thorne, R. M., and Angelopoulos, V.: Global distribution of wave amplitudes and wave normal angles of chorus waves using THEMIS wave observations, J. Geophys. Res., 116, A12205, doi:10.1029/2011JA017035, 2011.

Liu, K., Lemons, D. S., Winske, D., and Gary, S. P.: Relativistic electron scattering by electromagnetic ion cyclotron fluctuations: Test particle simulations, J. Geophys. Res., 115, A04204, doi:10.1029/2009JA014807, 2010.

Lyons, L. R. and Thorne, R. M.: Equilibrium structure of radiation belt electrons, J. Geophys. Res., 78, 2142-2149, 1973.

Lyons, L., Thorne, R., and Kennel, C.: Pitch-angle diffusion of radiation belt electrons within the plasmasphere, J. Geophys. Res., 77, 3455-3474, 1972

Ma, Q., Ni, B., Tao, X., and Thorne, R. M.: Evolution of the plasma sheet electron pitch angle distribution by whistler-mode chorus waves in non-dipole magnetic fields, Ann. Geophys., 30, 751760, doi:10.5194/angeo-30-751-2012, 2012.

MacDonald, W. M. and Walt, M.: Distribution function of magnetically confined electrons in a scattering atmosphere, Annals of
Physics, 15, 44-62, 1961.

Meredith, N., Horne, R., and Anderson, R.: Substorm dependence of chorus amplitudes: Implications for the acceleration of electrons to relativistic energies, J. Geophys. Res., 106, 1316513178, 2001.

Meredith, N. P., Horne, R. B., Thorne, R. M., and Anderson, R. R. Favored regions for chorus-driven electron acceleration to relativistic energies in the Earth's outer radiation belt, Geophys. Res. Lett., 30, 1871, doi:10.1029/2003GL017698, 2003.

Meredith, N. P., Horne, R. B., Glauert, S. A., Thorne, R. M., Summers, D., Albert, J. M., and Anderson, R. R.: Energetic outer zone electron loss timescales during low geomagnetic activity, J. Geophys. Res., 111, A05212, doi:10.1029/2005JA011516, 2006.

Meredith, N. P., Horne, R. B., Glauert, S. A., and Anderson, R. R. Slot region electron loss timescales due to plasmaspheric hiss and lightning-generated whistlers, J. Geophys. Res., 112, A08214, doi:10.1029/2007JA012413, 2007.

Meredith, N. P., Horne, R. B., Glauert, S. A., Baker, D. N., Kanekal, S. G., and Albert, J. M.: Relativistic electron loss timescales in the slot region, J. Geophys. Res., 114, A03222, doi:10.1029/2008JA013889, 2009a.

Meredith, N. P., Horne, R. B., Thorne, R. M., and Anderson, R. R.: Survey of upper band chorus and ECH waves: Implications for the diffuse aurora, J. Geophys. Res., 114, A07218, doi:10.1029/2009JA014230, 2009b.

Miyoshi, Y. S., Jordanova, V. K., Morioka, A., Thomsen, M. F., Reeves, G. D., Evans, D. S., and Green, J. C.: Observations and modeling of energetic electron dynamics during the October 2001 storm, J. Geophys. Res., 111, A11S02, doi:10.1029/2005JA011351, 2006.

$\mathrm{Ni}$, B. and Summers, D.: Resonance zones for electron interaction with plasma waves in the Earth's dipole magnetosphere: 2. Evaluation for oblique chorus, hiss, EMIC and magnetosonic waves, Phys. Plasmas, 17, 042903, doi:10.1063/1.3310835, 2010a.

$\mathrm{Ni}$, B. and Summers, D.: Resonance zones for electron interaction with plasma waves in the Earth's dipole magnetosphere: 1. Evaluation for field-aligned chorus, hiss and EMIC waves, Phys. Plasmas, 17, 042902, doi:10.1063/1.3310834, 2010b.

Ni, B., Thorne, R. M., Shprits, Y. Y., and Bortnik, J.: Resonant scattering of plasma sheet electrons by whistler-mode chorus: Contribution to diffuse auroral precipitation, Geophys. Res. Lett., 35 , L11106, doi:10.1029/2008GL034032, 2008.

Ni, B., Thorne, R. M., Horne, R. B., Meredith, N. P., Shprits, Y Y., Chen, L., and Li, W.: Resonant scattering of plasma sheet electrons leading to diffuse auroral precipitation: 1. Evaluation for electrostatic electron cyclotron harmonic waves, J. Geophys. Res., 116, A04218, doi:10.1029/2010JA016232, 2011a.

Ni, B., Thorne, R. M., Meredith, N. P., Horne, R. B., and Shprits, Y. Y.: Resonant scattering of plasma sheet electrons leading to diffuse auroral precipitation: 2. Evaluation for whistler mode chorus waves, J. Geophys. Res., 116, A04219, doi:10.1029/2010JA016233, 2011 b.

Ni, B., Thorne, R. M., Shprits, Y. Y., Orlova, K. G., and Meredith, N. P.: Chorus-driven resonant scattering of diffuse auroral electrons in nondipolar magnetic fields, J. Geophys. Res., 116, A06225, doi:10.1029/2011JA016453, 2011c.

Ni, B., Liang, J., Thorne, R. M., Angelopoulos, V., Horne, R B., Kubyshkina, M., Spanswick, E., Donovan, E. F., and Lummerzheim, D.: Efficient diffuse auroral electron scattering by 
electrostatic electron cyclotron harmonic waves in the outer magnetosphere: A detailed case study, J. Geophys. Res., 117, A01218, doi:10.1029/2011JA017095, 2011d.

O’Brien, T. P., Lorentzen, K. R., Mann, I. R., Meredith, N. P., Blake, J. B., Fennell, J. F., Looper, M. D., Milling, D. K., and Anderson, R. R.: Energization of relativistic electrons in the presence of ULF power and MeV microbursts: Evidence for dual ULF and VLF acceleration, J. Geophys. Res., 108, 1329, doi:10.1029/2002JA009784, 2003.

Orlova, K. G. and Shprits, Y. Y.: Dependence of pitch-angle scattering rates and loss timescales on the magnetic field model, Geophys. Res. Lett., 37, L05105, doi:10.1029/2009GL041639, 2010.

Orlova, K. G. and Shprits, Y. Y.: On the bounce-averaging of scattering rates and the calculation of bounce period, Phys. Plasmas, 18, 092904, doi:10.1063/1.3638137, 2011.

Roederer, J.: Dynamics of Geomagnetically Trapped Radiation, Springer, New York, 1970.

Schulz, M.: Particle motion and diffusion in geometric mirrors, Phys. Fluids, 19, 1835-1836, 1976.

Schulz, M.: Particle drift and loss rates under strong pitch angle diffusion in Dungey's model magnetosphere, J. Geophys. Res., 103, 61-67, 1998.

Schulz, M. and Chen, M.: Bounce-averaged Hamiltonian for charged particles in an axisymmetric but nondipolar model magnetosphere, J. Geophys. Res., 100, 5627-5635, 1995.

Schulz, M. and Lanzerotti, L. J.: Particle Diffusion in the Radiation Belts, Phys. Chem. Space, 7, 215 pp., Springer, New York, 1974.

Sheeley, B., Moldwin, M., Rassoul, H., and Anderson, R.: An empirical plasmasphere and trough density model: CRRES observations, J. Geophys. Res., 106, 25631-25641, 2001.

Shprits, Y. Y., Thorne, R. M., Horne, R. B., Glauert, S. A., Cartwright, M., Russell, C. T., Baker, D. N., and Kanekal, S. G.: Acceleration mechanism responsible for the formation of the new radiation belt during the 2003 Halloween solar storm, Geophys. Res. Lett., 33, L05104, doi:10.1029/2005GL024256, 2006a.

Shprits, Y. Y., Thorne, R. M., Horne, R. B., and Summers, D.: Bounce-averaged diffusion coefficients for fieldaligned chorus waves, J. Geophys. Res., 111, A10225, doi:10.1029/2006JA011725, 2006b.

Shprits, Y. Y., Elkington, S. R., Meredith, N. P., and Subbotin, D. A.: Review of modeling of losses and sources of relativistic electrons in the outer radiation belts: I. radial transport, J. Atmos. Sol. Terr. Phys., 70, 1679-1693, doi:10.1016/j.jastp.2008.06.008, 2008a.

Shprits, Y. Y., Subbotin, D. A., Meredith, N. P., and Elkington, S. R.:, Review of modeling of losses and sources of relativistic electrons in the outer radiation belts: II. local acceleration and loss, J. Atmos. Sol. Terr. Phys., 70, 1694-1713, doi:10.1016/j.jastp.2008.06.014, 2008b.

Shprits, Y. Y., Chen, L., and Thorne, R. M.: Simulations of pitch angle scattering of relativistic electrons with MLTdependent diffusion coefficients, J. Geophys. Res., 114, A03219, doi:10.1029/2008JA013695, 2009a.

Shprits, Y. Y., Subbotin, D., and Ni, B.: Evolution of electron fluxes in the outer radiation belt computed with the VERB code, J. Geophys. Res., 114, A11209, doi:10.1029/2008JA013784, 2009 b.

Shprits, Y. Y., Subbotin, D., Ni, B., Horne, R. B., Baker, D. N., and Cruce, P.: Profound change of the near-Earth radiation environment caused by solar superstorms, Space Weather, 9, S08007,
doi:10.1029/2011SW000662, 2011.

Su, Z., Zheng, H., and Wang, S.: Evolution of electron pitch angle distribution due to interactions with whistler mode chorus following substorm injections, J. Geophys. Res., 114, A08202, doi:10.1029/2009JA014269, 2009.

Su, Z., Xiao, F., Zheng, H., and Wang, S.: STEERB: A three-dimensional code for storm-time evolution of electron radiation belt, J. Geophys. Res., 115, A09208, doi:10.1029/2009JA015210, 2010a.

Su, Z., Zheng, H., and Wang, S.: Three-dimensional simulation of energetic outer zone electron dynamics due to wave-particle interaction and azimuthal advection, J. Geophys. Res., 115, A06203, doi:10.1029/2009JA014980, 2010b.

Subbotin, D., Shprits, Y., and Ni, B.: Three-dimensional VERB radiation belt simulations including mixed diffusion, J. Geophys. Res., 115, A03205, doi:10.1029/2009JA015070, 2010.

Subbotin, D. A., Shprits, Y. Y., and Ni, B.: Long-term radiation belt simulation with the VERB 3-D code: Comparison with CRRES observations, J. Geophys. Res., 116, A12210, doi:10.1029/2011JA017019, 2011.

Summers, D.: Quasi-linear diffusion coefficients for field-aligned electromagnetic waves with applications to the magnetosphere, J. Geophys. Res., 110, A08213, doi:10.1029/2005JA011159, 2005.

Summers, D. and Ni, B.: Effects of latitudinal distributions of particle density and wave power on cyclotron resonant diffusion rates of radiation belt electrons, Earth Planets Space, 60, 763-771, 2008.

Summers, D. and Thorne, R. M.: Relativistic electron pitchangle scattering by electromagnetic ion cyclotron waves during geomagnetic storms, J. Geophys. Res., 108, 1143, doi:10.1029/2002JA009489, 2003.

Summers, D., Thorne, R., and Xiao, F.: Relativistic theory of waveparticle resonant diffusion with application to electron acceleration in the magnetosphere, J. Geophys. Res., 103, 20487-20500, 1998.

Summers, D., Ni, B., and Meredith, N. P.: Timescales for radiation belt electron acceleration and loss due to resonant waveparticle interactions: 1. Theory, J. Geophys. Res., 112, A04206, doi:10.1029/2006JA011801, 2007a.

Summers, D., Ni, B., and Meredith, N. P.: Timescales for radiation belt electron acceleration and loss due to resonant wave-particle interactions: 2. Evaluation for VLF chorus, ELF hiss, and electromagnetic ion cyclotron waves, J. Geophys. Res., 112, A04207, doi:10.1029/2006JA011993, 2007b.

Summers, D., Ni, B., Meredith, N. P., Horne, R. B., Thorne, R. M., Moldwin, M. B., and Anderson, R. R.: Electron scattering by whistler-mode ELF hiss in plasmaspheric plumes, J. Geophys. Res., 113, A04219, doi:10.1029/2007JA012678, 2008.

Summers, D., Tang, R., and Thorne, R. M.: Limit on stably trapped particle fluxes in planetary magnetospheres, J. Geophys. Res., 114, A10210, doi:10.1029/2009JA014428, 2009.

Tao, X., Chan, A. A., Albert, J. M., and Miller, J. A.: Stochastic modeling of multidimensional diffusion in the radiation belts, J. Geophys. Res., 113, A07212, doi:10.1029/2007JA012985, 2008.

Tao, X., Albert, J. M., and Chan, A. A.: Numerical modeling of multidimensional diffusion in the radiation belts using layer methods, J. Geophys. Res., 114, A02215, doi:10.1029/2008JA013826, 2009. 
Tao, X., Bortnik, J., Albert, J. M., Liu, K., and Thorne, R. M.: Comparison of quasilinear diffusion coefficients for parallel propagating whistler mode waves with test particle simulations, Geophys. Res. Lett., 38, L06105, doi:10.1029/2011GL046787, 2011a.

Tao, X., Thorne, R. M., Li, W., Ni, B., Meredith, N. P., and Horne, R. B.: Evolution of electron pitch angle distributions following injection from the plasma sheet, J. Geophys. Res., 116, A04229, doi:10.1029/2010JA016245, 2011b.

Thorne, R. M.: Radiation belt dynamics: The importance of wave-particle interactions, Geophys. Res. Lett., 37, L22107, doi:10.1029/2010GL044990, 2010.

Thorne, R. M., O’Brien, T. P., Shprits, Y. Y., Summers, D., and Horne, R. B.: Timescale for $\mathrm{MeV}$ electron microburst loss during geomagnetic storms, J. Geophys. Res., 110, A09202, doi:10.1029/2004JA010882, 2005.

Thorne, R. M., Shprits, Y. Y., Meredith, N. P., Horne, R. B., Li, W., and Lyons, L. R.: Refilling of the slot region between the inner and outer electron radiation belts during geomagnetic storms, J. Geophys. Res., 112, A06203, doi:10.1029/2006JA012176, 2007.

Thorne, R. M., Ni, B., Tao, X., Horne, R. B., and Meredith, N. P.: Scattering by chorus waves as the dominant cause of diffuse auroral precipitation, Nature, 467, doi:10.1038/nature09467, 2010.

Varotsou, A., Boscher, D., Bourdarie, S., Horne, R. B., Glauert, S. A., and Meredith, N. P.: Simulation of the outer radiation belt electrons near geosynchronous orbit including both radial diffusion and resonant interaction with whistler-mode chorus waves, Geophys. Res. Lett., 32, L19106, doi:10.1029/2005GL023282, 2005.

Varotsou, A., Boscher, D., Bourdarie, S., Horne, R. B., Meredith, N. P., Glauert, S. A., and Friedel, R. H.: Three-dimensional test simulations of the outer radiation belt electron dynamics including electron-chorus resonant interactions, J. Geophys. Res., 113, A12212, doi:10.1029/2007JA012862, 2008.

Villalón, E. and Burke, W. J.: Pitch angle scattering of diffuse auroral electrons by whistler mode waves, J. Geophys. Res., 100, 19361-19369, 1995.
Wentworth, R. C.: Pitch angle diffusion a magnetic mirror geometry, Phys. Fluids, 6, 431-437, 1963.

Xiao, F., He, H., Zhou, Q., Zheng, H., and Wang, S.: Relativistic diffusion coefficients for superluminous (auroral kilometric radiation) wave modes in space plasmas, J. Geophys. Res., 111, A11201, doi:10.1029/2006JA011865, 2006.

Xiao, F., Su, Z., Zheng, H., and Wang, S.: Modeling of outer radiation belt electrons by multidimensional diffusion process, J. Geophys. Res., 114, A03201, doi:10.1029/2008JA013580, 2009a.

Xiao, F., Zong, Q.-G., and Chen, L.: Pitch-angle distribution evolution of energetic electrons in the inner radiation belt and slot region during the 2003 Halloween storm, J. Geophys. Res., 114, A01215, doi:10.1029/2008JA013068, 2009b.

Xiao, F., Su, Z., Chen, L., Zheng, H., and Wang, S.: A parametric study on outer radiation belt electron evolution by superluminous R-X mode waves, J. Geophys. Res., 115, A10217, doi:10.1029/2010JA015374, 2010a.

Xiao, F., Su, Z., Zheng, H., and Wang, S.: Three-dimensional simulations of outer radiation belt electron dynamics including cross $\odot \backslash$ diffusion terms, J. Geophys. Res., 115, A05216, doi:10.1029/2009JA014541, 2010b.

Xiao, F., Chen, L., and He, Y.: Bounce-averaged diffusion coefficients for superluminous wave modes in the magnetosphere, J. Atmos. Sol.-Terr. Phys., 73, 88-94, doi:10.1016/j.jastp.2009.12.012, 2011a.

Xiao, F., Chen, L., He, Y., Su, Z., and Zheng, H.: Modeling for precipitation loss of ring current protons by electromagnetic ion cyclotron waves, J. Atmos. Sol.-Terr. Phys., 73, 106-111, doi:10.1016/j.jastp.2010.01.007, 2011b.

Zong, Q.-G., Zhou, X.-Z., Wang, Y. F., Li, X., Song, P., Baker, D. N., Fritz, T. A., Daly, P. W., Dunlop, M., and Petersen, A.: Energetic electron response to ULF waves induced by interplanetary shocks in the outer radiation belt, J. Geophys. Res., 114, A10204, doi:10.1029/2009JA014393, 2009. 